\title{
Direkte Demokratie in der Schweiz - Länderbericht 2008/2009
}

\author{
Axel Tschentscher ${ }^{*}$
}

\section{Das Spektrum der Instrumente}

Über das Begriffsverständnis der direkten Demokratie, die Charakterisierung der einzelnen Instrumente und deren genaue Verteilung auf die Kantone gibt es ausführliche Analysen, ${ }^{1}$ so dass dieser Länderbericht ohne weitere Diskussion auf Einzelfragen von Volksinitiative und Referendum konzentriert bleibt. Hinsichtlich der Abstimmungspraxis wird es dabei zwangsläufig um exemplarische Vertiefung gehen müssen, denn in der Schweiz finden, so selbst jüngere Einschätzungen, weltweit summiert nach wie vor mehr als die Hälfte aller Volksabstimmungen statt. ${ }^{2}$

\section{Entwicklungen im Bund}

\section{a) Traditionelle Instrumente}

Im Bund bestehen seit Gründung des Bundesstaates (1848) das obligatorische Verfassungsreferendum und die Verfassungsinitiative auf Totalrevision, die traditionell auch den Kantonsverfassungen als Mindeststandard und Voraussetzung für die eidgenössische Gewährleistung vorgeschrieben waren und sind (Art. 6 Abs. 2 Bst. c BV $1848^{3}$, Art. 51 Abs. 1 Satz 2 BV $^{4}$ ). Erheblich ausgeweitet wurde die direkte Demokratie durch die erste Totalrevision der Bundesverfassung (1874), die ein Gesetzesreferendum einführte (Art. 89 BV 18745). Knapp zwei Jahrzehnte später (1891)

* Mein Dank gilt Frau Dominika Blonski, MLaw, für Recherchen, Korrekturen und inhaltliche Hinweise.

1 Beispielsweise Andreas Auer, Les droits politiques dans les cantons suisses, 1978, S. 6 ff., 34 ff.; Etienne Grisel, Initiative et référendum populaires. Traité de la démocratie semi-directe en droit suisse, 1987, S. 127 ff.; Alexander Trechsel/Uwe Serdült, Kaleidoskop Volksrechte. Die Institutionen der direkten Demokratie in den schweizerischen Kantonen (1970-1996), 1999, S. 1 ff.; Yvo Hangartner/Andreas Kley, Die demokratischen Rechte in Bund und Kantonen der Schweizerischen Eidgenossenschaft, 2000, Rn. 340; Michael Bützer, Direkte Demokratie in Schweizer Städten. Ursprung, Ausgestaltung und Gebrauch im Vergleich, 2007, S. 89 ff., 237 ff.

2 Adrian Vatter, Direkte Demokratie in der Schweiz: Entwicklungen, Debatten und Wirkungen, in: Markus Freitag/Uwe Wagschal (Hrsg.), Direkte Demokratie. Bestandsaufnahmen und Wirkungen im internationalen Vergleich, 2007, S. 71-113 (71).

3 Bundesverfassung der Schweizerischen Eidgenossenschaft vom 12. September 1848, in: Repertorium der eidgenössischen Tagsatzungen aus den Jahren 1814 bis 1848, Band 2, 1876, S. $764 \mathrm{ff}$.

4 Bundesverfassung der Schweizerischen Eidgenossenschaft vom 18. April 1999 (SR $101=$ www.admin.ch/ch/d/sr/1/101.de.pdf). Die offizielle Bezeichnung dieser Form lautet ,Eidgenössische Volksinitiative (Ausgearbeiteter Entwurf)".

5 Bundesverfassung der Schweizerischen Eidgenossenschaft vom 29. Mai 1874, BB1 1873 II 963, 1874 I 699 = www.amtsdruckschriften.bar.admin.ch. 
wurde dann neben die Volksinitiative auf Totalrevision die ungleich wirksamere Volksinitiative auf Partialrevision gestellt (Art. 121 BV 1874, „Volksanregung“). ${ }^{6}$ Sie war von Anfang an in den Formen eines ausgearbeiteten Entwurfs und einer allgemeinen Anregung möglich. Später hinzugekommen sind das fakultative Staatsvertragsreferendum (1921), ${ }^{7}$ das obligatorische Referendum für allgemein verbindliche Bundesbeschlüsse ohne Verfassungsgrundlage $(1949)^{8}$ und das obligatorische Referendum für den Beitritt zu internationalen Organisationen (1977). ${ }^{9}$

\section{b) Scheitern der allgemeinen Volksinitiative}

Die unattraktive Variante der allgemeinen Anregung sollte nach der Reform der Volksrechte (2003) durch eine ,allgemeinen Volksinitiative“ ersetzt werden, bei der die Bundesversammlung Gelegenheit erhält, das Anliegen sachgerecht auf Verfassungs- und Gesetzesstufe zu verteilen. Gleichzeitig sollte dieses neue direktdemokratische Instrument eine attraktive Alternative für die auf Bundesebene nicht eingeführte Gesetzesinitiative darstellen. Seine Einführung ist aber inzwischen gescheitert. Schon der vom Bundesrat vorgeschlagene, attraktivitätssteigernde Bonus, der darin gelegen hätte, dass für eine allgemeine Volksinitiative nur 70.000 statt 100.000 Unterschriften genügen, wurde in der Reform der Volksrechte ignoriert. Vor allem aber bedurfte es weitergehender Gesetzesbestimmungen, um zu verhindern, dass eine vom Volk angenommene allgemeine Volksinitiative wegen Uneinigkeit von National- und Ständerat dann doch nicht umgesetzt werden kann. Auch der Rechtsweg bei inhaltlich unbefriedigender Umsetzung sollte als neue Beschwerde ,wegen Missachtung von Inhalt und Zweck einer allgemeinen Volksinitiative durch die Bundesversammlung" (Art. 189 Abs. 1bis BV n.F. ${ }^{10}$ ) auf Gesetzesstufe ausgestaltet werden. Darum wurde die Reform der Volksrechte zunächst nicht vollständig in Kraft gesetzt, sondern nur hinsichtlich der unkomplizierten Erweiterung des Staatsvertragsreferendums (Art. 141 Abs. 1 Bst. d Ziff. 3 BV ${ }^{11}$ ). Als dann der Entwurf für ein Bundesgesetz über die Einführung der allgemeinen Volksinitiative so kompliziert und praxisuntauglich geriet, dass das Parlament darauf überhaupt nicht eintrat (2006), wurde von der Staatspolitischen Kommission des Nationalrats

6 Bundesbeschluß betreffend Revision der Bundesverfassung vom 8. April 1891, BB1 1891 II $330=$ www.amtsdruckschriften.bar.admin.ch.

7 Eidgenössische Volksinitiative ,für die Unterstellung von unbefristeten oder für eine Dauer von mehr als 15 Jahren abgeschlossenen Staatsverträgen unter das Referendum (Staatsvertragsreferendum)“; Übersicht dazu unter www.admin.ch/ch/d/pore/vi/vis11.html.

8 Eidgenössische Volksinitiative „Rückkehr zur direkten Demokratie“; siehe .../vi/vis56.html.

9 Bundesbeschluss über die Neuordnung des Staatsvertragsreferendums: Volksinitiative „gegen die Beschränkung des Stimmrechts bei Staatsverträgen mit dem Ausland“, Gegenentwurf; siehe .../vi/vis108.html.

10 Bundesbeschluss über die Änderung der Volksrechte vom 4. Oktober 2002, AS 20031949 $(1951)=$ www.admin.ch/ch/d/as/2003/1949.pdf.

11 Bundesbeschluss über das Inkrafttreten der direkt anwendbaren Bestimmungen der Änderung der Volksrechte vom 4. Oktober 2002 vom 19. Juni 2003, AS $20031953=$ =../as/2003/1953. pdf. 
die Rückänderung der Verfassung vorgeschlagen. Diesem Vorschlag haben sich Bundesrat, Kantone und Parteien inzwischen angeschlossen, so dass voraussichtlich das Instrument der allgemeinen Volksinitiative vor seiner Inkraftsetzung bereits wieder durch Verfassungsrevision abgeschafft wird. Die entsprechende Beschlussvorlage ist bereits ausgearbeitet und dürfte angesichts der inzwischen einhelligen Meinung zum Scheitern der allgemeinen Volksinitiative wohl auch angenommen werden. ${ }^{12}$

\section{c) Diskussionen um ein Finanzreferendum}

Im Bund gibt es, anders als in den Kantonen, ${ }^{13}$ bisher kein Finanzreferendum. Seine Einführung wurde früher durch Volksabstimmung abgelehnt $(1956)^{14}$ und anlässlich der Totalrevision der Bundesverfassung zwar in den Entwurf integriert (1996), ${ }^{15}$ dann aber in der Reform der Volksrechte nicht übernommen. Zu einem obligatorischen Verfassungsreferendum in Finanzfragen kommt es nur, wenn verfassungskräftige Detailbestimmungen zu Steuern geändert werden, welche beispielsweise bei der Mehrwertsteuer wegen der Höchstsatzklausel sehr konkret ausfallen (Art. 130 Abs. 1 BV). Im Übrigen besteht finanzverfassungsrechtlich zur Ausgabenbegrenzung nur das Instrument der Schuldenbremse (Art. 126 BV). Dessen beschränkte Wirksamkeit hat dazu geführt, dass die Staatspolitische Kommission des Nationalrats jüngst eine parlamentarische Initiative zur Einführung des Finanzreferendums nach Anhörung verschiedener Experten unterstützte (2006). ${ }^{16}$ Wegen der verfassungsrechtlichen Öffnungsklausel, nach der Bundesbeschlüsse durch Gesetz dem Referendum unterstellt werden können (Art. 141 Abs. 1 Bst. c BV), wäre dies sogar ohne Verfassungsänderung möglich. Das Vorhaben fand allerdings in der Bundesversammlung trotz Verlängerung des Geschäfts keine ausreichende Unterstützung, so dass es abgeschrieben wurde (2008). Ein Grund für die außergewöhn-

12 Entwurf vom 19. Dezember 2008 zu einem Bundesbeschluss über den Verzicht auf die Einführung der allgemeinen Volksinitiative, in: BB1 200913 = www.admin.ch/ ch/d/ff/2009/13. pdf. Zu weiteren Gründen des Scheiterns siehe Martin Graf, Die ,,allgemeine Volksinitiative“ - ein Lehrstück missglückter Rechtsetzung, in: leges 2007, S. 343-354 (344 ff.); Nadja Braun, Gescheiterte allgemeine Volksinitiative: Sind die Volksrechte reformträge?, in: leges 2007, S. 337-342 (338 ff.). Frühe Skepsis gegenüber einer solchen Einheitsinitiative bei Pierre Tschannen, Stimmrecht und politische Verständigung. Beiträge zu einem erneuerten Verständnis von direkter Demokratie, 1995, S. $471 \mathrm{ff}$.

13 Zum kantonalen Finanzreferendum siehe unten S. 210.

14 Botschaft des Bundesrates an die Bundesversammlung über das Ergebnis der Volksabstimmung vom 30. September 1956 betreffend die Bundesbeschlüsse über die Revision der Brotgetreideordnung des Landes und das Volksbegehren betreffend Ausgabenbeschlüsse der Bundesversammlung, in: BB1 1956 II 657 = www.amtsdruckschriften.bar.admin.ch.

15 Vgl. Botschaft über eine neue Bundesverfassung vom 20. November 1996, Geschäft Nr. 96.091, S. 76, 466 ff. = www.parlament.ch/ d / suche / seiten / geschaefte.aspx ?gesch_id $=199-60091$.

16 Übersicht zum Geschäft Nr. 03.401 - Parlamentarische Initiative: Einführung eines Finanzreferendums unter .../geschaefte.aspx?gesch_id=20030401. 
liche Zurückhaltung dürfte darin liegen, dass mit einem Finanzreferendum auf Bundesebene die öffentlich sehr umstrittenen Rüstungsausgaben direkt anfechtbar würden. ${ }^{17}$

\section{Entwicklungen in den Kantonen und Gemeinden}

\section{a) Traditionelle Instrumente}

Sowohl Landsgemeinde als auch Rousseau - das ist die Antwort, die von der neueren verfassungsgeschichtlichen Forschung auf die Frage nach den Wurzeln der direkten Demokratie gegeben wird. ${ }^{18}$ Denn einerseits lässt sich, bei allen Unterschieden zum modernen Demokratieverständnis, ${ }^{19}$ eine mentale Kontinuität der versammlungsdemokratisch geprägten politischen Kultur in den Landsgemeinden seit dem Spätmittelalter diagnostizieren. ${ }^{20}$ Mit dem föderativen Referendum in den zugewandten Orten und den Volksanfragen in den Städterepubliken waren sogar außerhalb der Landsgemeinden erste Wurzeln für gemeinschaftliche Mitbestimmung vorhanden. ${ }^{21}$ Andererseits war die kantonale Entwicklung durch die Montagnard-Verfassung und den Girondisten-Entwurf des Jahres 1793 der französischen Revolution inspiriert, die insoweit beide auf die Ideen des Genfer Philosophen zurückführen. ${ }^{22}$ Die historischen Entwicklungsschübe der 1830er und 1860er Jahre nahmen ihren Anfang in den Kantonen und mündeten 1848 und 1874 jeweils in eine Bundesverfassung. Die Dynamik der kantonalen Innovation wird deutlich, wenn man sich vergegenwärtigt, dass in dieser Zeitspanne (1830 bis 1873) alle Kantonsverfassungen außer derjenigen von Appenzell Innerrhoden eine Totalrevision erfuhren und insgesamt sechs sogar viermal vollständig neu erlassen wurden. ${ }^{23}$ Bis heute sind die direktdemokratischen Instrumente auf der Kantonsebene jeweils früher und weiter ausgebaut als auf der Bundesebene. So kannten bereits sechs Regenerationsver-

17 Vgl. dazu das Geschäft Nr. 06.442 - Parlamentarische Initiative: Mitsprache des Volkes bei Militärausgaben unter .../geschaefte.aspx?gesch_id=20060442.

18 Beispielsweise Hangartner/Kley, Demokratische Rechte (Fn. 1), Rn. 1287 ff.; Benjamin Adler, Die Entstehung der direkten Demokratie. Das Beispiel der Landsgemeinde Schwyz 1789-1866, 2006, S. 11.

19 Zu diesen René Pahud de Mortanges, Schweizerische Rechtsgeschichte. Ein Grundriss, 2007, S. $65 \mathrm{f}$.

20 Vgl. Gustav Vogt, Referendum, Veto und Initiative in den neueren schweizerischen Kantonsverfassungen, in: Zeitschrift für die gesamte Staatswissenschaft 29 (1873), S. 350-380 (363); speziell für den Kanton Schwyz Adler, Entstehung (Fn. 18), S. 140.

21 Adrian Vatter, Kantonale Demokratien im Vergleich. Entstehungsgründe, Interaktionen und Wirkungen politischer Institutionen in den Schweizer Kantonen, 2002, S. $228 \mathrm{ff}$.

22 Alfred Kölz, Neuere schweizerische Verfassungsgeschichte, Band 1: Ihre Grundlinien vom Ende der Alten Eidgenossenschaft bis 1848, 1992, S. 315 f., 469 ff.; im Ergebnis ebenso bereits Reto Caratsch, Die Initiative zur Verfassungsrevision, 1926, S. 33 f., 56, 115 ff.; Riccardo Jagmetti, Der Einfluss der Lehren von der Volkssouveränität und vom pouvoir constituant auf das schweizerische Verfassungsrecht, 1920, S. 7 ff., 112.

23 Tabellarische Übersicht bei Vogt, Referendum (Fn. 20), S. 358 f. 
fassungen (1830/1831) neben dem unumstrittenen Instrument des obligatorischen Verfassungsreferendums auch die Verfassungsinitiative des Volkes. ${ }^{24}$ Gleichzeitig steigerte der Kanton St. Gallen sein Konzept der Volkssouveränität zu einem Volksveto gegen Gesetze, das in der demokratischen Bewegung der 1860er Jahre dann zum Vorbild für das Gesetzesreferendum werden sollte. ${ }^{25}$ Dieses Instrument unterlag allerdings dem „Vetoprinzip“, nach dem nur die absolute Mehrheit der stimmberechtigten Gemeindeeinwohner das Veto für diese Gemeinde bedeutete und im Kanton nur das absolute Mehr der gewichteten Gemeindestimmen - ausgedrückt zudem innerhalb von 45 Tagen nach Erlass des Gesetzes - dieses insgesamt zu Fall bringen konnte. Im Ergebnis wurden dadurch die nicht abgegebenen Stimmen als stillschweigende Annahme gewürdigt, was beispielsweise dazu führte, dass ein Veto aus dem Jahr 1855 scheiterte, obwohl $71 \%$ der abgegebenen Stimmen für das Veto waren. ${ }^{26}$ Wegen der hohen Hürden gelang es dem Volk unter der St. Galler Regenerationsverfassung (1831 bis 1861) bei 40 Versuchen nur viermal (letztmalig 1834), die Anwendung eines Gesetzes zu verhindern. ${ }^{27}$ Das Instrument der Gesetzesinitiative fand sich 1845 zunächst im Kanton Waadt, der gleichzeitig auch das (jeweils parlamentarisch veranlasste) plebiszitäre Gesetzesreferendum einführte. ${ }^{28}$ Nicht durchgesetzt hat sich die periodische Volksbefragung der Genfer Verfassung von 1847, nach dem bis zur Abschaffung des Instruments (1993) dem Volk alle 15 Jahre die Totalrevision der Verfassung vorgeschlagen werden musste.

\section{b) Erweitertes Referendum}

Neben dem obligatorischen Verfassungsreferendum und der Verfassungsinitiative auf Gesamtrevision, die bereits durch die Bundesverfassung als Mindeststandard vorgeschrieben sind (Art. 51 Abs. 1 Satz 2 BV), ${ }^{29}$ finden sich in den Kantonen regelmäßig auch die Verfassungsinitiative für Partialrevisionen, das Gesetzesreferen-

24 Alfred Kölz, Die Bedeutung der Französischen Revolution, in: Andreas Auer (Hrsg.), Les origines de la démocratie directe en Suisse, 1996, S. 105-117 (109); ders., Neuere schweizerische Verfassungsgeschichte, Band 2: Ihre Grundlinien in Bund und Kantonen seit 1848, 2004, S. $199 \mathrm{f}$.

25 Kölz, Bedeutung (Fn. 24), S. 109 f.; ders., Verfassungsgeschichte I (Fn. 22), S. 309 ff., 316; weitere Details bei Vogt, Referendum (Fn. 20), S. 368 ff.; Urs Dietschi, Das Volksveto in der Schweiz. Ein Beitrag zur Geschichte der Volksgesetzgebung, 1926, S. 50 ff.; Vatter, Kantonale Demokratien (Fn. 21), S. 233 ff. mit Tabelle S. 238.

26 Zahlen in der Tabelle bei Dietschi, Volksveto (Fn. 25), S. 70.

27 Dietschi, Volksveto (Fn. 25), S. 65 ff. (67 ff.); Vatter, Kantonale Demokratien (Fn. 21), S. 241, mit Angaben zu weiteren Vetokantonen auf S. 246.

28 Kölz, Bedeutung (Fn. 24), S. $110 \mathrm{f}$.

29 So die herrschende Meinung; einzelne Stimmen in der Literatur sehen in Art. 51 BV bereits eine Pflicht zur Einführung der Verfassungsinitiative auf Partialrevision. Zum Meinungsstand siehe Hangartner/Kley, Demokratische Rechte (Fn. 1), Rn. 1364. 
dum sowie das Verwaltungs- und das Finanzreferendum. ${ }^{30}$ Der Form nach können Dekrete, Verordnungen, Konkordate und sogar kantonale Stellungnahmen im eidgenössischen Vernehmlassungsverfahren der kantonalen Volksabstimmung unterworfen werden. ${ }^{31}$ Der Art nach kann ein Referendum fakultativ oder obligatorisch sein; selbst das obligatorische Gesetzesreferendum ist geläufig (z.B. Art. 69 Abs. 1 Satz $2 \mathrm{KV}-\mathrm{GL}) .^{32}$ Dem Inhalt nach kann ein obligatorisches Referendum beispielsweise für die Genehmigung der Benutzung des Untergrunds oder für Wasserrechtsverleihungen vorgesehen sein. Mehrere Kantone kennen obligatorische Volksabstimmungen zu Stellungnahmen ihres Kantons über den Bau von Kernkraftwerken, Aufbereitungsanlagen oder Lagerstätten für radioaktive Abfälle (z.B. Art. 52 Nr. 5 KV-NW, Art. 32 Bst. f KV-SH). ${ }^{33}$ In den Kantonen Bern und Nidwalden gibt es zudem das Instrument des „Volksvorschlags“, bei dem das Volk einen Gegenvorschlag zu einem Gesetz oder einem Grundsatzbeschluss des Parlaments einbringt (Art. 63 Abs. $3 \mathrm{KV}-\mathrm{BE}$, Art. 54 a Abs. $3 \mathrm{KV}-\mathrm{NW}$ ). ${ }^{34}$ Außergewöhnlich sind auch die weitergehenden Mitspracherechte der Landsgemeinde. So wird im Kanton Glarus der Steuerfuß direkt vom Volk festgesetzt (Art. 69 Abs. 2 Bst. e KV-GL). Erwähnenswert ist schließlich die im Vorgriff auf die Referendumsmöglichkeit bestehende Ingerenz der „Volksdiskussion“, bei der einzelne Bürgerinnen oder Bürger bereits in die Beratung des Kantonsrats eingreifen können (Art. $56 \mathrm{KV}$-AR). ${ }^{35}$

Das Finanzreferendum ist inzwischen in allen Kantonen eröffnet. ${ }^{36}$ Einzige Ausnahme war bis zur kantonalen Verfassungsrevision (2003) der Kanton Waadt. Gerade beim kantonalen Referendum in Finanzfragen geht die schweizerische Demokratie weit über die in Deutschland auf Landesebene etablierte Volksbeteiligung hinaus, denn dort herrschen traditionell Finanzausschlussklauseln vor, ${ }^{37}$ und selbst die Volksinitiative ist durch Haushaltsvorbehalte eingeschränkt. ${ }^{38}$ In der Schweiz ist

30 Einen Überblick bis zum Stand 1997 bieten Georg Lutz/Dirk Strohmann, Wahl und Abstimmungsrecht in den Kantonen. Droits politique dans les cantons, Bern u.a. 1998, S. 139 ff.; Trechsel/Serdült, Kaleidoskop (Fn. 1), S. 123 ff.

31 Übersicht bei Trechsel/Serdült, Kaleidoskop (Fn. 1), S. 15.

32 Verfassung des Kantons Glarus von 1988 (SR 131.217 = .../sr/1/131.217.de.pdf).

33 Verfassung des Kantons Unterwalden nid dem Wald (Nidwalden) von 1965 (SR 131.216.2 $=\ldots / \mathrm{sr} / 1 / 131.216 .2$.de.pdf); Verfassung des Kantons Schaffhausen von 2002 (SR 131.223 = ... sr/1/131.223.de.pdf) - letztere zusätzlich auch für Stellungnahmen zur Aufnahme von Straßen in das Nationalstraßennetz: Art. 32 Bst. g KV-SH.

34 Verfassung des Kantons Bern (SR 131.212 = .../sr/1/131.212.de.pdf).

35 Verfassung des Kantons Appenzell Ausserrhoden von 1995 (SR 131.224.1 = .../sr/1/131.224. 1.de.pdf).

36 Überblick zum Ausgabenreferendum und anderen Formen des Finanzreferendums bei Hangartner/Kley, Demokratische Rechte (Fn. 1), Rn. 1814 ff., 1938 ff.

37 Vergleichende Bestandsaufnahme bei Christian Waldhoff, Finanzwirtschaftliche Entscheidungen in der Demokratie - ein Verfassungsvergleich zwischen der Schweiz und Deutschland zum Freiheitsschutz des Bürgers vor staatlicher Finanzmacht, in: Martin Bertschi u.a. (Hrsg.), Demokratie und Freiheit, 1999, S. 181-227 (191 ff., 204 ff.).

38 Vgl. zur häufig problematischen Anwendung solcher Klauseln Christoph Degenhart, Direkte Demokratie auf Bundesebene nach dem Grundgesetz - Anmerkungen zu einem Gesetzentwurf, in: Klaus Stern/Klaus Grupp (Hrsg.), GS Joachim Burmeister, 2005, S. 87-99 (94 f.); Fabian Wittreck, Direkte Demokratie und Verfassungsgerichtsbarkeit. Eine kritische Übersicht zur 
demgegenüber das Referendum über Finanzfragen zunächst indirekt als Teil des Gesetzesreferendums möglich, wenn nämlich das Gesetz selbst die Finanzierung regelt oder wenn es, obwohl selbst ohne Finanzvorschriften, wegen der resultierenden Mehrkosten inhaltlich vom Volk abgelehnt wird. Darüber hinaus gibt es das eigentliche Finanzreferendum, das sich als zusätzliches Instrument auf die Finanzbeschlüsse des Parlaments richtet, sofern sie bestimmte Kreditlimiten überschreiten - mithin auf „Erlasse, die ausschließlich aufgrund ihres Finanzcharakters dem Referendum unterstellt werden. ${ }^{\text {"39 }}$ Sachgerechterweise wird hier mit unterschiedlichen Limiten für einmalige und wiederkehrende Ausgaben gearbeitet. Außerdem gibt es das Finanzreferendum in der obligatorischen und in der fakultativen Variante, wobei etwa die Hälfte der Kantone beide Formen für je unterschiedliche Kreditvolumina kombiniert. Bei wiederkehrenden Ausgaben steht das fakultative Referendum in mehreren Kantonen bereits ab einem Finanzvolumen von 50.000 Franken zur Verfügung.

\section{c) Erweiterte Volksinitiative}

Das Verfahren der Volksinitiative ist in den Kantonen anders ausgestaltet und das Instrument regelmäßig weitergehend als im Bund. ${ }^{40}$ So gibt es beispielsweise nicht überall die Pflicht, eine Rückzugsklausel auf dem Unterschriftenbogen anzubringen. Auch beginnt in einigen Kantonen das Verfahren mit einer blossen Anmeldung oder informellen Vorprüfung statt mit einer obligatorischen Vorprüfung. In Zürich genügt für die Ungültigerklärung einer Volksinitiative nicht die einfache Mehrheit, sondern es ist ein Quorum von zwei Dritteln der anwesenden Kantonsratsmitglieder verlangt (Art. $28 \mathrm{KV}-\mathrm{ZH}$ ). Vor allem variieren die Werte zur Zahl der erforderlichen Unterschriften, der Sammelfrist und der Behandlungsfristen. Die Einzelinitiative, also eine Volksinitiative von nur einer einzigen Person, ist inzwischen zur seltenen Ausnahme geworden. Ansonsten gelten in den kleinsten Kantonen Unterschriftenzahlen von 300-600, in den größten solche zwischen 10.000 und 15.000 (Zürich neu 6.000), was insgesamt einem Spektrum von etwa $2 \%$ bis $5 \%$ der Stimmberechtigten entspricht (Bund: 100.000 entsprechend $2 \%$ ). Die Sammelfristen bewegen sich typischerweise in einem Spektrum von drei bis sechs Monaten (Bund: 18 Monate).

Im Gegensatz zum Bund kennen die Kantone neben der Verfassungsinitiative sämtlich eine Gesetzesinitiative. Einzelne kantonale Initiativrechte richten sich darüber hinaus als administrative Initiativen auf den Erlass von Verordnungen oder Dekreten oder auf die Ein- oder Abberufung einer Behörde. Das als „Volksvorschlag" bezeichnete Instrument in Bern und Nidwalden ${ }^{41}$ betrifft allerdings kein administratives Handeln und ist auch nicht im engeren Sinne ein Initiativrecht, son-

deutschen Verfassungsrechtsprechung in Fragen der unmittelbaren Demokratie von 2000 bis 2002, in: JöR 53 (2005), S. 111-185 (117 ff., 121 ff., 136 ff., 144 f., 165 ff.) mit dem Fazit der Entbehrlichkeit solcher Klauseln: S. 183.

39 Trechsel/Serdült, Kaleidoskop (Fn. 1), S. 37.

40 Überblick bei Lutz/Strohmann, Wahl und Abstimmungsrecht (Fn. 30), S. 139 ff.

41 Siehe dazu oben bei Fn. 34. 
dern eine aktive Zusatzoption beim Gesetzesreferendum. Wie ein parlamentarisches Initiativrecht wirkt indes die (ähnlich klingende) „Volksmotion“ oder der „Volksauftrag"; damit verlangt eine kleine Gruppe Stimmberechtigter (100 bis 300) analog zur parlamentarischen Motion eines Abgeordneten die verbindliche Beauftragung der Kantonsregierung zur Ausarbeitung eines Gesetzes (z.B. Art. $34 \mathrm{KV}-\mathrm{SO}){ }^{42}$ In Zürich ist sogar die Einzelinitiative (Individualinitiative) an das Kantonsparlament möglich. ${ }^{43}$ Durch „Memorialsantrag“ kann eine einzelne stimmberechtigte Person das Programm der Landsgemeinde (Memorial) mitbestimmen und so auch die Teilrevision der Verfassung auslösen (z.B. Art. 138 Abs. 3 KV-GL). Die im Bund gescheiterte ,allgemeine Volksinitiative“, die eine flexible Zuordnung der Materie zur Verfassungs- oder Gesetzesstufe ermöglicht, findet sich als „Einheitsinitiative“ auf kantonaler Ebene realisiert (Art. $53 \mathrm{KV}$-AR).

Eine weitere Sonderkonstellation besteht in der Initiative auf Auslösung des bundesrechtlichen Kantonsreferendums. Dieses Recht steht acht Kantonen gemeinsam zu (Art. 141 Abs. 1 BV), beruht aber auf der Einzelentscheidung jedes Kantons, die regelmäßig durch dessen Parlament getroffen wird. In einigen Kantonen wird diese Befugnis dem Kantonsvolk vorbehalten: als kantonale Volksinitiative auf Erhebung des eidgenössischen Kantonsreferendums. ${ }^{44}$ Vereinzelt ist zudem die vom Bundesrecht vorgesehene Standesinitiative, mit der ein einzelner Kanton die Beratung der Bundesversammlung auslösen kann (Art. 161 Abs. 1 BV), im Gewand eines kantonalen direktdemokratischen Instruments an das Volk weitergereicht, mithin eine kantonale Volksinitiative auf Einreichung einer eidgenössischen Standesinitiative (z.B. Art. 12 Abs. 2 Nr. 2 KV-GR).

\section{Direkte Demokratie im Bund}

In diesem ersten Länderbericht sei vorab ein Überblick über die Dokumentation und über das Verfahren der Volksinitiative gegeben.

\section{Der Legislativprozess und seine Dokumentation}

\section{a) Publikationsorgane des Bundes}

Die Systematische Sammlung des Bundesrechts (SR) bildet den Endpunkt der Gesetzesdokumentation und dessen alltagspraktische Referenz. Sie enthält das geltende Bundesrecht in der jeweils bereinigten und nach Sachgebieten sortierten Fassung. Die Sammlung ist vollständig im Internet einsehbar (www.admin.ch/ch/d/sr/) und

42 Verfassung des Kantons Solothurn von 1986 (SR 131.221 = .../sr/1/131.221.de.pdf). Dazu ausführlich Hangartner/Kley, Demokratische Rechte (Fn. 1), Rn. 383 ff., 2396 ff.

43 Details bei Trechsel/Serdült, Kaleidoskop (Fn. 1), S. 413 ff.

44 Beispielsweise die Verfassung des Kantons Zug von 1894 in $§ 41$ Bst. r KV-ZG (SR 131.218 $=\ldots /$ sr/1/131.218.de.pdf). 
wird dort, im Gegensatz zur gedruckten Fassung, laufend nachgeführt (Art. 14 Abs. 2 PublV 45 ).

Die Amtliche Sammlung des Bundesrechts (AS) ist das eigentliche, die Rechtswirkungen der Publikation auslösende Instrument des Bundes. ${ }^{46}$ Sie gilt in Konfliktfällen mit Vorrang vor allen anderen Publikationen (vgl. Art. 8 PublG). Die dort aufgeführten chronologischen Änderungen sind für Publikationszeitpunkte ab dem 1. September $1998 \mathrm{im}$ Internet einsehbar (www.admin.ch/ch/d/as/), vorher nur in der Druckversion. Die Erlasse erscheinen allerdings erst dann in der Amtlichen Sammlung, wenn der Zeitpunkt des Inkrafttretens bestimmt ist, mithin nach dem Referendumsprozess. Eine Ausnahme bilden die als dringlich erklärten Bundesgesetze (Art. 165 Abs. 1 BV), die zwar, wenn ihre Geltungsdauer ein Jahr übersteigt, ebenfalls dem Referendum unterstehen (Art. 141 Abs. 1 Bst. b BV), aber sofort, das heißt vor dem Referendumsprozess, in Kraft gesetzt werden. ${ }^{47}$

Damit bleibt das Bundesblatt (BBl) als relevantes Medium für die Dokumentation des direktdemokratischen Beteiligungsverfahrens selbst. Es steht ab dem Juni 1999 als reguläre Internetausgabe (www.admin.ch/ch/d/ff/) und für die Zeit davor (1849-1999) als durchsuchbares Faksimile im Schweizerischen Bundesarchiv zur Verfügung (www.amtsdruckschriften.bar.admin.ch). Im Bundesblatt werden die Verfügungen der Bundeskanzlei über die Vorprüfung, das Zustandekommen oder das Scheitern von Volksinitiativen und Referenden veröffentlicht. Auch bieten die abgedruckten Botschaften des Bundesrates an die Bundesversammlung, die Ablehnungsbeschlüsse der Bundesversammlung mit (seltenen) direkten Gegenentwürfen sowie die detaillierten Abstimmungsergebnisse eine zeitnahe Erläuterung.

\section{b) Sonstige amtliche Informationen}

Noch übersichtlicher, aber nicht mehr in Gestalt einer gesetzlich garantierten Publikation, informiert die Bundeskanzlei auf ihren Aktualitätsseiten über hängige Volksinitiativen (www.bk.admin.ch/aktuell/abstimmung/vi/) und Referenden (.../abstimmung/ref/), sowie auf ihren Themenseiten über eingereichte Volksinitiativen (www.bk.admin.ch/themen/pore/vi/), eingereichte Referenden (.../pore/ref/) und den Verlauf von Volksabstimmungen insgesamt (.../pore/va/) - jeweils zurückgehend bis zum Anfang des Bundesstaates (1848) bzw. bis zur Einführung des Gesetzesreferendums (1874) und der „Volksanregung“ (1891). Von hier aus lassen sich über die „Detailangaben zur Volksinitiative“ auch die indirekten Gegenentwürfe erschließen, die das Parlament mit seiner Ablehnungsempfehlung verbindet. Sie sind

45 Verordnung über die Sammlungen des Bundesrechts und das Bundesblatt vom 17. November 2004 (Publikationsverordnung, PublV, SR 170.512.1 = .../sr/1/170.512.1.de.pdf).

46 Details dazu im Bundesgesetz über die Sammlungen des Bundesrechts und das Bundesblatt vom 18. Juni 2004 (Publikationsgesetz, PublG, SR 170.512 = .../sr/1/170.512.de.pdf), insbesondere Art. 2-10 PublG.

47 Siehe beispielsweise das Gesetz über die Änderung des Bundesgesetzes über die Banken und Sparkassen (Bankengesetz, BankG) vom 19. Dezember 2008 (Verstärkung des Einlegerschutzes), AS $200955=. . . /$ as/2009/55.pdf. 
über ihre eigene Dossiernummer und diejenige unter „Konnexe Geschäfte“ in der Geschäftsdatenbank der Bundesversammlung (Curia Vista) einsehbar. Diese Datenbank umfasst unter anderem die Wortprotokolle von Nationalrat und Ständerat (Druckfassung: Amtliches Bulletin, AB), so dass sich die Argumentation inzwischen sehr detailliert im Internet nachvollziehen lässt. Nicht öffentlich sind allerdings die Kommissionsdebatten. Die Einsichtnahme in deren Wortprotokolle kann im Einzelfall zum Zweck einer wissenschaftlichen Auswertung bei den Parlamentsdiensten beantragt werden. Für die noch nicht abgeschlossenen Volksinitiativen und Referenden empfehlen sich die Dossierseiten des Parlaments, auf denen die jeweils aktuellsten Beratungen und Beschlüsse für die Medien zusammengestellt sind (www.parlament.ch). Kantonale Volksabstimmungen lassen sich außer über die jeweiligen Kantonsseiten auch durch die gemeinsame Plattform von Bund und Kantonen erschließen (www.ch.ch).

\section{c) Nichtamtliche Informationsquellen}

Die wichtigste politikwissenschaftliche Auswertung der eidgenössischen Volksabstimmungen findet sich als „VOX-Analyse“ in einer Kurzfassung im Internet (www. polittrends.ch/vox-analysen) und lässt sich inhaltlich erheblich umfangreicher in Form eines gedruckten Hefts pro Abstimmungstermin bestellen. Brauchbare Hintergrundinformationen zum Verlauf der Diskussion bieten auch die Tagespresse unmittelbar nach den Abstimmungsterminen (z.B. www.nzz.ch) sowie das Fernsehen auf seinen Themenseiten (tagesschau.sf.tv/hintergrund/abstimmungen) und komprimiert auf seinen Auslandsseiten (www.swissinfo.ch/ger/politik_schweiz/abstimmungen). Über diese nichtamtlichen Internetseiten wird teilweise auch amtliches Informationsmaterial erschlossen, das zwar auf Behördenservern existiert, dort aber nicht mehr rekonstruierbar erschlossen ist (z.B. Kartenmaterial zu den Abstimmungen unter www.bfs.admin.ch/bfs/portal/de/index/themen/17.html).

\section{Die Volksinitiative im Bund - Verfahrensgang und politischer Prozess}

Im direktdemokratischen Instrumentarium des Bundes ist die formulierte Volksinitiative auf Teilrevision der Verfassung das wirkmächtigste Instrument (Art. 139 BV). Im Bund gehen die Volksinitiativen regelmäßig auf eine Teilrevision der Verfassung, weil das Instrument einer Gesetzesinitiative (im Unterschied zur Kantonsebene) hier nicht verwirklicht ist. Die formulierte Volksinitiative ist gegenüber der allgemeinen Anregung beliebter, weil bei ihr die Gefahr geringer ausfällt, dass die Bundesversammlung Inhalt oder Ziele in der Umsetzung verfälscht. Im Gegensatz zum Referendum ist die Initiative nicht bloß reagierend, sondern aktiv mitgestaltend - sie hat das größte „Inputpotential“ aller direktdemokratischen Instrumente. ${ }^{48}$ Die-

48 Ralph Kampwirth, Volksentscheid und Öffentlichkeit. Anstöße zu einer kommunikativen Theorie der direkten Demokratie, in: Theo Schiller (Hrsg.), Direkte Demokratie in Theorie und kommunaler Praxis, 1999, S. 17-68 (21). 
ser Effekt tritt sogar dann ein, wenn die Initiative formell betrachtet ohne Erfolg bleibt, denn über indirekte Gegenvorschläge des Parlaments wird ebenfalls die Richtung der Gesetzgebung beeinflusst. Eine Volksinitiative kann darum ihren rechtspolitischen Zielvorstellungen nahe kommen, selbst wenn sie ihr formuliertes Verfassungsziel nicht ganz erreicht. Der mittelbare Effekt ist besonders deshalb wichtig, weil sich der direkte Erfolg nur selten einstellt: insgesamt gelingt auf Bundesebene weniger als $10 \%$ der Initiativen die Annahme. ${ }^{49}$ Schließlich ist die Volksinitiative auch attraktiv, weil sie die Initianten als zentrale Akteure der Politik ins Gespräch bringt. Meist sind es die bei Wahlen oder im Parlament unterlegenen Parteien, die auf diese Weise um Aufmerksamkeit für sich und für die Besonderheiten ihrer politischen Überzeugung werben - obgleich gerade deren Erfolgsaussichten geringer sind als diejenigen der im Parlament dominanten Parteien. ${ }^{50}$

\section{a) Initiierungsphase}

Ein Initiativkomitee muss die Initiative vor Beginn der Unterschriftensammlung zur Vorprüfung bei der Bundeskanzlei einreichen (Art. $69 \mathrm{BPR}^{51}$ ). Die Vorprüfung, deren Ergebnis in einer anfechtbaren Verfügung eröffnet wird, erstreckt sich nicht auf den Inhalt der Volksinitiative, sondern lediglich auf Form und Übersetzungskorrektheit sowie auf den Titel der Initiative, der von der Bundeskanzlei geändert werden kann, wenn er irreführend ist, Werbung enthält oder die Gefahr einer Verwechslung begründet. Durch eine vorbehaltlose Rückzugsklausel auf dem Unterschriftsbogen wird sichergestellt, dass keine Volksabstimmungen über obsolet gewordene Initiativen stattfinden (Art. 68 Abs. 1 Bst. c und Art. 73 BPR)..$^{52}$ Die geprüfte und nötigenfalls formell korrigierte Initiative wird im Bundesblatt veröffentlicht, womit die Sammelfrist von 18 Monaten zu laufen beginnt (Art. 71 BPR). Vor Ablauf der Frist holt das Initiativkomitee bei den Wohngemeinden die Stimmrechtsbescheinigungen ein (Art. 19 VPR $^{53}$ ). Hierbei kam es in der Vergangenheit gelegentlich zu Irritationen, wenn eine Initiative bei der Sammlung relativ knapp über der Mindestgrenze von 100.000 Unterschriften lag und die kantonalen Behörden nur mit Verzögerung die Bescheinigungen ausstellen konnten, so dass ein knappes Scheitern drohte. Zur formellen Einreichung werden die Unterschriften insgesamt der Bundeskanzlei

49 Es waren dies bisher, auffällig gehäuft in den letzten Jahrzehnten, die folgenden 16 Initiativen (mit Abstimmungsjahr): Schächtverbot (1893), Absinthverbot (1908), Proporzwahl (1918), Spielbankenverbot (1920), Staatsvertragsreferendum (1921), Kursaalspiele (1928), Demokratiestärkung (1949), Preismissbrauchsverhinderung (1982), Moorschutz (1987), Atomkraftwerksmoratorium (1990), 1. August (1993), Alpentransitverkehr(1994), UNO-Beitritt (2002), Verwahrung (2004), Gentechnik (2005), Unverjährbarkeit (2008).

50 Vatter, Kantonale Demokratien (Fn. 21), S. 279 f., 295.

51 Bundesgesetz über die politischen Rechte vom 17. Dezember 1976 (SR 161.1 = .../sr/1/161.1. de.pdf).

52 Zur Ratio dieses ,rigiden“ Systems auf Bundesebene Etienne Grisel, Initiative et référendum populaires. Traité de la démocratie semi-directe en droit suisse, 1987, S. $137 \mathrm{ff}$.

53 Verordnung über die politischen Rechte vom 24. Mai 1978 (SR 161.11 = .../sr/1/161.11.de. pdf). 
übergeben, was politisch häufig in Form einer öffentlichkeitswirksamen Aktion mit Presseeinladung zur Information vor dem Bundeshaus verbunden wird. Die Bundeskanzlei stellt die ausreichende Zahl gültiger Unterschriften in einer Verfügung über das Zustandekommen fest, die ebenfalls im Bundesblatt veröffentlicht wird. Die Initiierungsphase ist damit abgeschlossen.

\section{b) Botschafts- und Beratungsphase}

Eine zustande gekommene Volksinitiative wird zunächst dem Bundesrat zugeleitet, der in einer Botschaft an die Bundesversammlung feststellt, ob die Initiative gültig ist, ob er selbst sie zur Annahme empfiehlt oder ob, falls dies nicht der Fall ist, ein Gegenentwurf der Bundesversammlung ratsam erscheint. In aller Regel konzentriert sich, weil Bundesrat und Bundesversammlung fast immer gegen die Annahme der Initiative votieren, die folgende Diskussion auf deren Gültigkeit und auf die Frage eines Gegenentwurfes. Zu einem förmlichen Gegenvorschlag, der den Stimmberechtigten zusammen mit der Initiative und einer Stichfrage vorgelegt wird (Art. 76 BPR, Art. 101 f. ParlG ${ }^{54}$ ), kommt es nur selten. Häufiger versucht die Bundesversammlung, der Initiative den politischen Wind aus den Segeln zu nehmen, indem sie die Arbeiten an einem Gesetz beginnt, das den inhaltlichen Anliegen der Initianten jedenfalls teilweise Rechnung trägt. In solchen Fällen spricht man, weil der politische Konnex zur Initiative deutlich erkennbar ist, von einem indirekten Gegenentwurf. Für die Gültigerklärung, die durch einfachen Bundesbeschluss hinsichtlich der gesamten Initiative oder hinsichtlich eines Teils von ihr erfolgt, sind die beiden Kammern des Parlaments an einen sehr engen Katalog von Kriterien gebunden. Nur wenn es an der Einheit der Form oder Materie fehlt oder wenn die Initiative gegen zwingendes Völkerrecht verstößt, ist die Ungültigerklärung vorgesehen (Art. 194 Abs. 2 und 3 BV). Ein ungeschriebener Ungültigkeitsgrund ist zudem die offensichtliche Undurchführbarkeit einer Initiative. Besonders umstritten waren dabei in jüngerer Zeit die Fälle deutlich erkennbarer, aber einfacher Völkerrechtswidrigkeit. ${ }^{55}$

Die Behandlungsfristen bei Initiativen sind teils überlagernd, teils addieren sie sich. ${ }^{56}$ Der Bundesrat muss eine zustande gekommene Initiative auf Teilrevision spätestens ein Jahr nach der Einreichung mit einer Botschaft und dem Entwurf eines Bundesbeschlusses an die Bundesversammlung weiterleiten, bei einem Gegenvorschlag innerhalb von 18 Monaten (Art. 97 ParlG). Die Bundesversammlung beschließt innerhalb von 30 Monaten nach Einreichung der Initiative über die Empfehlung und den Gegenentwurf (ordentliche Behandlungsfrist, Art. 100 f. ParlG). Sie

54 Bundesgesetz über die Bundesversammlung vom 13. Dezember 2002 (Parlamentsgesetz, ParlG, SR $171.10=\ldots /$ sr/1/171.10.de.pdf).

$55 \mathrm{Zu}$ dieser Grundsatzfrage unten S. 219.

56 Jeweils aktuelle Beispiele für den Fristverlauf: .../vi/vis_1_3_1_5.html. 
kann diese Frist um ein weiteres Jahr hinausschieben, wenn in engem sachlichen Zusammenhang mit der Volksinitiative beraten wird (Art. 105 Abs. 1 ParlG). ${ }^{57}$

\section{c) Abstimmungsphase}

Hat die Bundesversammlung entschieden, so unterbreitet der Bundesrat die Initiative grundsätzlich innerhalb der nächsten zehn Monate dem Volk zur Abstimmung; ausnahmsweise darf die Abstimmung innerhalb von zehn Monaten nach Ablauf der parlamentarischen Behandlungsfrist erfolgen. Eine zustande gekommene Volksinitiative kann folglich im Extremfall erst mehr als vier Jahre später zur Abstimmung kommen (52 Monate). Rechnet man die Sammlungsfrist (18 Monate) und die häufig ein Jahr vor der Unterschriftensammlung beginnende öffentliche Diskussion hinzu, so kann eine einzelne Initiative bis zu sieben Jahre lang auf der Agenda der Bundespolitik stehen.

Als mögliche Abstimmungstermine des Bundes sind vier Sonntage im Jahr reserviert, die sich nach den Feiertagen richten (Art. 2 a VPR) und typischerweise im Februar/März, Mai/Juni, September und November liegen. Alle vier Jahre, wenn im Oktober die Gesamterneuerungswahl des Nationalrats stattfindet, fällt der Septembertermin aus. ${ }^{58}$ Die Abstimmungstermine werden in der Regel auch von Kantonen und Gemeinden für ihre eigenen Volksabstimmungen genutzt, obgleich dies mit dem Nachteil einer geteilten Aufmerksamkeit verbunden ist.

\section{d) Umsetzungsphase}

Die Initiative auf Änderung der Bundesverfassung hat Erfolg, wenn sie in der Volksabstimmung das doppelte Mehr von Volk und Ständen erringt (Art. 140 Abs. 1 Bst. a BV). Das Volksmehr ist die Mehrheit der Stimmenden. Das Ständemehr ist die Mehrheit der Stände, wobei jeweils die Mehrheit des Volkes in einem Kanton als Standesstimme zählt. Die sechs kleinen, früher als „Halbkantone“ bezeichneten Kantone (Obwalden, Nidwalden, Basel-Stadt, Basel-Landschaft, Appenzell Ausserrhoden und Appenzell Innerrhoden) haben - im Gegensatz zu den 20 „Vollkantonen“ - je eine halbe Standesstimme. Entsprechend charakterisiert man das Ergebnis mit zwei Angaben für die Mehrheit (z.B.: 56,2 \% Ja-Stimmen, 19 5/2 Stände ${ }^{59}$ ). Einen knappen Erfolg erringt man demgemäß mit 50,1 \% Ja-Stimmen und 10 4/2 Ständen.

Ist die Volksinitiative angenommen, so tritt sie sofort als Änderung der Bundesverfassung in Kraft. Es bedarf allerdings häufig einer Umsetzung im Gesetzesrecht. Wegen der eingeschränkten Durchsetzbarkeit des Verfassungsvorrangs gegenüber

57 Ein Beispiel dafür war die Verwahrungsinitiative (Erläuterung unten bei Fn. 95), die erst knapp vier Jahre nach ihrer Einreichung zur Abstimmung kam.

58 Vorausberechnung der nächsten Jahre: .../va/vab_1_3_3_1.html.

59 Ergebnis der Verwahrungsinitiative, unten Fn. $9 \overline{5}$. 
Bundesgesetzen (Art. $190 \mathrm{BV}$ ) sind die Initianten von diesem Umsetzungsprozess abhängig, was schon mehrfach zu Unstimmigkeiten geführt hat. ${ }^{60}$

\section{Grundsätzliche Fragen}

\section{a) Primat der direkten Demokratie}

Zu den Charakteristika der direkten Demokratie in der Schweiz gehört ihre prinzipielle Unumstrittenheit. Hier gibt es einen deutlichen Kontrast zu der Fundamentalkritik, der die direkte Demokratie in Deutschland immer noch ausgesetzt ist. ${ }^{61}$ Dabei sind die Probleme - geringe Abstimmungsbeteiligung, populistische Inhalte, fehlende politische Führung, knappe (Zufalls-)Ergebnisse, laienhafte Verfassungsund Strafrechtsfortbildung, überproportionaler Einfluss organisierter Interessengruppen, verlangsamte Gesetzgebungsprozesse und vieles andere ${ }^{62}-$ in der Schweiz dieselben wie überall, doch die Volksrechte treffen gleichwohl nirgends auf grundsätzliche Ablehnung. Im Gegenteil: selbst bei knappsten Abstimmungsresultaten wird die Legitimität im Ergebnis nicht bezweifelt und jeder Kritiker einer undemokratischen Einstellung geziehen; selbst bei geringsten Abstimmungsbeteiligungen (im Bund regelmäßig unter $50 \%$, in den Gemeinden sehr häufig unter $20 \%$ ) nimmt das politische Gemeinwesen die Entscheidung als eine gemeinsame an; selbst komplizierteste Erweiterungen der Volksrechte können auf eine breite Zustimmung zählen, und umgekehrt werden selbst wohlwollend gemeinte Regulierungen des Abstimmungskampfes als Beschränkung der politischen Freiheit empfunden und mit deutlicher Mehrheit abgelehnt. ${ }^{63}$ Der Primat des unmittelbar erklärten Volkswillens

60 Als Beispiele dafür siehe die Unverjährbarkeits- und die Verwahrungsinitiative, unten S. 226.

61 Detailanalyse bei Wittreck, Direkte Demokratie (Fn. 38), S. 134 f., 141 ff., 153 ff., 163 f., 173 ff.; zu den vergleichsweise hohen Hürden in Deutschland Degenhart, Direkte Demokratie (Fn. 38), S. 87 ff.; Fabian Wittreck, Direkte Demokratie vor Gericht. oder: Direkte Demokratie und Verfassungsgerichtsbarkeit - Ein gestörtes Verhältnis, in: Hermann K. Heußner/Otmar Jung (Hrsg.), Mehr direkte Demokratie wagen, 2. Aufl., 2009, S. 397-415 (403 ff.); zum Unterschied zwischen Deutschland und der Schweiz außerdem Udo Steiner, Schweizer Verhältnisse in Bayern? - Zu Bürger- und Volksbegehren im Freistaat, 2000, S. 26 f.

62 Früh mit Einzelaspekten Max Imboden, Helvetisches Malaise, 1964, S. 6 ff., 19 ff. Umfassender dann Raimund E. Germann, Politische Innovation und Verfassungsreform. Ein Beitrag zur schweizerischen Diskussion über die Totalrevision der Bundesverfassung, 1975, S. 146 ff.; ders., Staatsreform. Der Übergang zur Konkurrenzdemokratie, 1994, S. 18 ff., 194 ff.; Walter Wittmann, Direkte Demokratie. Bremsklotz der Revitalisierung, 2001, S. 23 ff. Hinterfragung gängiger Kritik bei Tschannen, Stimmrecht (Fn. 12), S. 257 ff.; Gebhard Kirchgässner/ Lars P. Feld/Marcel R. Savioz, Die direkte Demokratie. Modern, erfolgreich, entwicklungsund exportfähig, 1999, S. 20 ff., 60 ff., 71 ff., 165 ff.; Vatter, Direkte Demokratie (Fn. 2), S. 87 ff. Historische Schuldzuweisung des Veltlin-Verlusts an die Langsamkeit des Referendums bei Vogt, Referendum (Fn. 20), S. 366 f.

63 Vgl. dazu die Volksabstimmung über die sogenannte Maulkorbinitiative unten S. 225. Ein historisches Beispiel bietet der Kampf gegen den Gebrauch dringlicher Bundesbeschlüsse als Instrument des Notrechts zwischen 1934 und 1938; siehe dazu David Reich, Direkte Demokra- 
über repräsentative politische Willensbildung ist unumstritten. Selbst wenn Volkswille auf (rechtsstaatlich geronnenen) Volkswillen stößt, etwa weil die direkte Demokratie des Kantons an den Grenzen der Bundesverfassung oder des Völkerrechts scheitert, wird dies von vielen als eine unangemessene Beschränkung der Demokratie empfunden. ${ }^{64}$ Der Bürgerschaft ist die direkte Demokratie geradezu heilig wahrscheinlich auch darum, weil sie in Kantonen und Bund Instrument um Instrument gegen die jeweils dominierende Regierungselite erkämpft werden musste. ${ }^{65}$ Nach wie vor gilt in der Schweiz der Satz, dass eine Verfassung ,um so beständiger ist, je rückhaltloser sie dem Volkswillen freies Spiel lässt." "66 Man kann einen einzelnen Entscheid des Volkes dezidiert falsch (im Sinne von schlecht) finden, beispielsweise die Ablehnung des EWR-Beitritts im Dezember 1992, und man kann auch die gegenwärtige Praxis der Bundesbehörden im Umgang mit den Volksrechten kritisieren, ${ }^{67}$ aber man kann hierzulande niemanden hören, der die legislative Souveränität des Volkes an ein Parlament abtreten möchte.

\section{b) Völkerrechtswidrige Volksinitiativen}

Seit Jahren wird eine Reformdiskussion um die Ausweitung der Gültigkeitsanforderungen für Volksinitiativen geführt. Bisher zieht nämlich nur ein Verstoß gegen zwingendes Völkerrecht (ius cogens) die Ungültigerklärung nach sich, wofür es lediglich einen Beispielsfall gibt: Die SD-Initiative (Volksinitiative ,für eine vernünftige Asylpolitik"; neuer Art. 69quater BV; eingereicht am 6. Juli 1992; ungültig erklärt am 14. März 1996; www.admin.ch/ch/d/pore/vi/vis223.html) hatte eine ausnahmslose sofortige Wegweisung aller illegal eingereisten Asylbewerberinnen und -bewerber gefordert, was gegen das Non-Refoulement-Prinzip verstoßen und im Einzelfall zu Folter und Tod geführt hätte. Einfache Völkerrechtswidrigkeit hindert die Volksabstimmung hingegen nicht. So verstieß die Verwahrungsinitiative, mit der die Haftüberprüfung von Sexualstraftätern beschränkt werden sollte, gegen die Europäische Menschenrechtskonvention, aber nicht gegen Bestimmungen des zwingenden Völkerrechts. Demgemäß konnte die Initiative nicht für ungültig erklärt werden, musste dem Volk zur Abstimmung vorgelegt werden und wurde dann, was politisch als Widerspruch erschien, wegen der Völkerrechtsbindung doch nicht wirksam umgesetzt. Ein ähnlicher Mangel in der wortgetreuen Umsetzung war schon früher bei

tie in der Krise. Die Funktion des Notrechts in der Schweiz während Weltwirtschaftskrise und Zweitem Weltkrieg dargestellt am Beispiel des Warenhausbeschlusses 1933-1945, 2007, S. $202 \mathrm{ff}$.

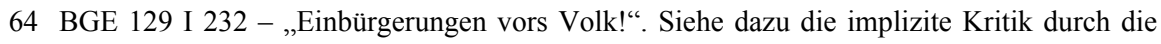
Einbürgerungsinitiative unten S. 223.

65 Kölz, Verfassungsgeschichte II (Fn. 24), S. 32 ff.; ders., Der demokratische Aufbruch des Zürchervolkes. Eine Quellenstudie zur Entstehung der Zürcher Verfassung von 1869, 2000, S. 11 ff.; Adler, Entstehung (Fn. 18), S. 181.

66 Vogt, Referendum (Fn. 20), S. 362.

67 Betont Andreas Auer, Les institutions de la démocratie directe en Suisse: une lente dégradation, in: leges 2004/3, S. 35-48 (43 ff.). 
der Alpeninitiative aufgetreten und wird mit den immer häufiger völkerrechtskritischen Anliegen mehr und mehr vorkommen. Will man in solchen Fällen die erfolgreiche Volksinitiative nicht gleichzeitig als ein Mandat zum Völkerrechtsbruch verstehen, so ergibt sich für die verfassungskonkretisierenden und rechtsanwendenden Behörden eine Pflichtenkollision, die in der Schweiz gegenwärtig zugunsten des Völkerrechts entschieden wird. ${ }^{68}$

Auf der Basis des geltenden Verfassungsrechts lässt sich das Auseinanderfallen von Gültigerklärung und Wirksamkeit einer solchen Initiative mit der bisherigen Praxis nicht vermeiden. Der Widerspruch wird als nächstes bei der Minarettinitiative virulent werden, während bei der Ausschaffungsinitiative analog zur erwähnten SD-Initiative eine Ungültigerklärung nahe liegt. ${ }^{69}$ Der Ständerat hat die Gültigerklärung der völkerrechtswidrigen Minarettinitiative nur bei etlichen Gegenstimmen beschlossen (24 zu 16 Stimmen). Damit ist der Weg zum Abstimmungskampf eröffnet, der ungeachtet des Ausgangs der Volksabstimmung bereits im Vorfeld zu internationalen Missverständnissen und Anfeindungen führen könnte.

Trotz dieser Befürchtungen hat das Parlament richtigerweise die Ungültigkeitsgründe nicht entgegen der ausdrücklichen Verfassungsregelung (Art. 139 und 194 BV) und langjährigen Verfassungspraxis eigenmächtig ausgeweitet. Die schweizerische Bundesverfassung kennt bisher keine Ewigkeitsklausel. Materielle Schranken der Verfassungsrevision werden zwar seit langem erwogen, ${ }^{70}$ bestehen aber nach geltendem Recht nur in sehr engen Grenzen. Allzu trickreich wäre es, den Begriff des zwingenden Völkerrechts nunmehr landesrechtlich statt völkerrechtlich auszufüllen - also als „,schweizerisches“ ius cogens. ${ }^{71}$ Ebenso unlauter wäre die Ausdehnung der einzigen anerkannten ungeschriebenen Schranke der faktischen Undurch-

68 Walter Kälin, Internationale Menschenrechtsgarantien als Schranke der Revision von Bundesverfassungsrecht. Das Beispiel völkerrechtswidriger Asylinitiativen, in: AJP 1993, S. 243-257 (254 ff.); Yvo Hangartner, Völkerrecht und schweizerisches Landesrecht, in: Walter R. Schluep (Hrsg.), FS Arnold Koller, 1993, S. 651-679 (676); Andreas Auer/Bénédicte Tornay, Aux limites de la souveraineté du constituant: l'initiative „Pour des naturalisations démocratiques“, in: AJP 2007, S. 740-747 (743 ff.); Regina Kiener/Melanie Krüsi, Bedeutungswandel des Rechtsstaats und Folgen für die (direkte) Demokratie am Beispiel völkerrechtswidriger Volksinitiativen, in: ZBl. 2009, S. 237-257 (253 f.). Vage indes Hangartner/ Kley, Demokratische Rechte (Fn. 1), Rn. 507 ff. (561). Als völkerrechtswidrige Initiativen, die gleichwohl für gültig erklärt wurden, zählt Robert Baumann, Völkerrechtliche Schranken der Verfassungsrevision, in: ZB1 2007, S. 181-210 (191 ff.), auf (mit Einreichungsjahr): RheinauInitiative (1953), Überfremdungsinitiativen (1965, 1969, 1972, 1974), Staatsvertragsinitiative (1973), Armeeabschaffungsinitiativen (1986, 1999), Alpeninitiative (1990), Verkehrshalbierungsinitiative (1996), Sonntagsinitiative (1998), Volksrechteinitiative (1999), Verwahrungsinitiative (2000), Tierschutzinitiative (2003) und Einbürgerungsinitiative (2005). Die Gründe der Völkerrechtswidrigkeit finden sich bei Kälin, Menschenrechtsgarantien, a.a.O., S. $253 \mathrm{ff}$.

69 Vgl. die Kriterien bei Kälin, Menschenrechtsgarantien (Fn. 68), S. 255 ff. (257). A.A. Johannes Reich, Direkte Demokratie und völkerrechtliche Verpflichtungen im Konflikt. Funktionellrechtlich differenzierte Herstellung praktischer Konkordanz zwischen der Beachtung des Völkerrechts und konfligierenden Volksinitiativen im schweizerischen Bundesverfassungsrecht, in: ZaöRV 68 (2008), S. 979-1025 (1016 ff.), der auch diese Initiative für gültig hält.

70 Überblick m.w.N. bei Hangartner/Kley, Demokratische Rechte (Fn. 1), Rn. 483 ff.

71 Überblick m.w.N. bei Baumann, Völkerrechtliche Schranken (Fn. 68), S. 189. 
führbarkeit auf die Tatbestände einer politischen oder rechtlichen Unmöglichkeit. ${ }^{72}$ Damit bleibt als aufrichtige Lösung nur eine Änderung der Bundesverfassung. Diskutiert wird beispielsweise, die Europäische Menschenrechtskonvention als einen Mindeststandard festzulegen, der auch gegenüber Verfassungsänderungen Bestand hat. Eine solche Reform wäre allerdings der Sache nach eine hierzulande abgelehnte Ewigkeitsklausel, die zudem inhaltlich sehr weit geht, weil sie im praktischen Ergebnis die jeweilige Auslegung des Europäischen Gerichtshofs für Menschenrechte über das Initiativrecht des schweizerischen Volkes stellt. Insgesamt bleibt es dabei, dass die Frage des sachgerechten Umgangs mit Volksbegehren, die einfachem Völkerrecht widersprechen, bisher unbeantwortet bleibt. ${ }^{73}$

Abschließend sei noch auf einen Wertungswiderspruch in der staatsrechtlichen Praxis hingewiesen, der nicht die Gültigkeitskriterien, sondern die Umsetzung von völkerrechtswidrigen Initiativen betrifft. In den beiden Fällen, in denen eine völkerrechtswidrige Initiative bisher angenommen wurde, der Alpeninitiative (1994) und der Verwahrungsinitiative (2004), hat der Bundesrat die völkerrechtskonforme Umsetzung zulasten der initiativgetreuen Umsetzung gewählt. Unter zwei gleichermaßen auf Verfassungsebene festgelegten Pflichten hat er sich damit zugunsten der Völkerrechtsbindung entschieden und so dem ungeschriebenen schweizerischen Grundsatz „Völkerrecht bricht Landesrecht“ Rechnung getragen. Gleichzeitig verfolgt das Bundesgericht mit der sogenannten Schubert-Praxis eine entgegengesetzte Richtung: Obwohl für das Gericht gleichzeitig Bundesgesetze und Völkerrecht maßgebend sind (Art. 190 BV), wird, wenn der Gesetzgeber den Verstoß gegen einen (älteren) Staatsvertrag bewusst in Kauf genommen hat, einseitig dem Bundesgesetz der Vorrang eingeräumt. ${ }^{74}$ Würde man die Schubert-Praxis auf die Alpeninitiative und die Verwahrungsinitiative übertragen, so müsste das Völkerrecht dort ebenso zurücktreten, weil Volk und Stände den Verstoß gegen völkerrechtliche Bindungen auch in diesen Fällen bewusst in Kauf genommen haben. ${ }^{75}$ Es müsste sogar erst

72 So aber André Grisel, A propos de la hiérarchie des normes juridiques, in: ZB1 1987, S. 377-395 (386f.): ,impossibilité purement juridique“, ,impossibilité de droit“, ,incompatibilité manifeste, qui ne laisse aucune place à un léger doute“. Vgl. zur Argumentation Hangartner/ Kley, Demokratische Rechte (Fn. 1), Rn. 497 ff.

73 Pierre Tschannen, Wem gehört die Verfassung? Neuer Streit um die Gewaltenteilung, in: ZBJV 143 (2007), S. 793-806 (801).

$74 \mathrm{Zu}$ Genese, Begründungsschwäche und Reichweite der Schubert-Praxis Walter Kälin, Der Geltungsgrund des Grundsatzes „Völkerrecht bricht Landesrecht“, in: Guido Jenny/Walter Kälin (Hrsg.), FG Schweizerischer Juristentag, 1988, S. 45-65 (60 ff.); aktuelle Hinweise z.B. bei René Rhinow/Markus Schefer, Schweizerisches Verfassungsrecht, 2. Aufl., 2009, Rn. 3637 ff.; Pierre Tschannen, Staatsrecht der Schweizerischen Eidgenossenschaft, 2. Aufl., 2007, § 9 Rn. 33 f.

75 Baumann, Völkerrechtliche Schranken (Fn. 68), S. 198 f., 210. A.A., weil das Volk insoweit nie „,bewußt“ entscheide: Roger Nobs, Volksinitiative und Völkerrecht. Eine Studie zu Volksinitiativen im Kontext der schweizerischen Aussenpolitik unter besonderer Berücksichtigung des Verhältnisses zum Völkerrecht, 2006, S. 363; Kiener/Krüsi, Bedeutungswandel (Fn. 68), S. 251. Zur argumentativen Schwäche der Schubert-Praxis insbesondere Kälin, Geltungsgrund (Fn. 74), S. 63: „Es ist widersprüchlich, eine generelle Bindung ... an das spätere Gesetz (rich- 
recht zurücktreten, weil erstens dem unmittelbaren Volksentscheid eine stärkere demokratische Legitimation zukommt als dem Bundesgesetz der volksgewählten Repräsentanten und zweitens die vom Volk geänderte Verfassung im Normenrang höher als das Bundesgesetz steht. Das ist allerdings nicht die einzige Möglichkeit, den Wertungswiderspruch in der staatsrechtlichen Praxis zu beseitigen. - Die zweite bestünde darin, die Schubert-Praxis aufzugeben, wofür die jüngste Rechtsprechung des Bundesgerichts erste Anhaltspunkte bietet. ${ }^{76}$

\section{c) Verfassungstext und materielle Verfassung}

Ein rechtsästhetisches, teils sogar rechtssystematisches Problem der Volksinitiative auf Bundesebene ist ihr Durchgriff auf den Verfassungstext. Schon unter der alten Bundesverfassung hatte die Einfügung von formellen Verfassungsbestimmungen, die nicht zur materiellen Verfassung gehören, zu einer großen Unübersichtlichkeit des Verfassungsdokuments beigetragen - etwa mit dem Absinthverbot von 1908 (Art. 32 b BV 1874). Die neue Bundesverfassung hatte zum Beginn des neuen Jahrhunderts eine übersichtliche Neuordnung geschaffen. Wegen der Volksinitiative gelangen nun aber wieder Detailregelungen in den Verfassungstext hinein, die eigentlich nicht verfassungswürdigen Inhalts sind und so zur Zerfaserung der Verfassung führen. Beispielsweise wurden mit der Verwahrungs- und Unverjährbarkeitsinitiative einzelne Regelungen, die in das Strafgesetzbuch gehört hätten, als neue Artikel eingefügt (Art. 123 a und 123 b BV). Ohne eine Ergänzung des direktdemokratischen Instrumentariums um eine attraktive Gesetzesinitiative wird sich dieser Prozess nicht stoppen lassen. ${ }^{77}$

\section{d) Reformdiskussionen}

Trotz allgemeiner Zustimmung zur direkten Demokratie als solcher wird fortlaufend eine Diskussion über Reformen geführt - allerdings meist als Detaildiskussion auf der Basis des bestehenden Instrumentariums. Nur selten werden, praktisch ohne Erfolgsaussicht, grundlegendere Reformen gefordert. ${ }^{78}$

Unter den Detailänderungen ist der bedingte Rückzug von Initiativen bereits weitgehend konsentiert. Bei dem bisher allein möglichen unbedingten Rückzug kann nämlich für die Initianten ein Dilemma entstehen, wenn das Parlament zwar einen ausgearbeiteten indirekten Gegenvorschlag erlässt, dieser aber bei anderen Bevölkerungsgruppen so unbeliebt ist, dass dagegen wiederum ein erfolgreiches Referendum droht. Zukünftig soll darum dem Initiativkomitee eine goldene Brücke zum

tigerweise) abzulehnen, sie aber für den Fall bewusster Abweichung vom Völkerrecht zu bejahen."

76 BGE 133 I 270 E. 1.2.2 S. 274 - Fristenstillstand (einschränkungsloser Vorrang der EMRK gegenüber dem neuen Bundesgerichtsgesetz).

77 Vgl. Graf, Die ,allgemeine Volksinitiative“ (Fn. 12), S. 346 f.

78 Z.B. bei Wittmann, Direkte Demokratie (Fn. 62), S. 157 ff. 
Rückzug gebaut werden, indem sie diesen davon abhängig machen dürfen, dass der indirekte Gegenvorschlag des Parlaments tatsächlich in Kraft tritt. Auslöser und erster Anwendungsfall dieser Reform wird die Volksinitiative „Lebendiges Wasser" der Fischer und Umweltschützer sein (Renaturierungsinitiative), deren Zielsetzung das Parlament durch Gesetzesänderungen entsprechen will, was wiederum dem Interesse der Energiewirtschaft entgegenläuft.

Viel diskutiert wurde in der Vergangenheit die krasse Erfolgswertungleichheit der Stimmen bei Doppelmehr-Abstimmungen. Eine Volksinitiative oder das obligatorische Referendum bei Verfassungsrevisionen benötigt neben dem Volksmehr auch das Ständemehr. Der Kleinstkanton Appenzell Innerrhoden erhält dabei eine halbe Standesstimme, während den Stimmberechtigten im Kanton Zürich insgesamt nur eine (ganze) Standesstimme zukommt. Im Ergebnis genießen die Stimmberechtigten in Kleinkantonen dadurch ein bis zu 40-faches Stimmgewicht. Berechnet man den theoretischen Fall einer optimalen Kombination von Kleinkantonen, deren Standesstimmen zusammen gerade genügen, um eine Doppelmehr-Abstimmung zum Scheitern zu bringen, so kommt man zu einer kleinsten theoretischen Sperrminorität die unter $10 \%$ der schweizerischen Stimmberechtigten liegt. ${ }^{79}$ Das Problem dieses Ungleichgewichts schien sich zu verschärfen, als die Doppelmehr-Abstimmungen seit den 70er Jahren geradezu inflationsartig zunahmen. Doch ist die theoretische Sperrminorität wenig aussagekräftig, weil die kantonalen Allianzen sich real anders bilden. Als kritische Fälle bleiben nur diejenigen Abstimmungen übrig, bei denen eine Kollision von Volks- und Ständemehr in der Form eintritt, dass die Kantone mehrheitlich mit Nein stimmen, während das Volk insgesamt zustimmt. Und solche Fälle sind sehr selten, wenn sie auch in jüngerer Zeit zunehmen. ${ }^{80}$ Zwischen 1848 und 1969 gab es nur zwei, seit 1970 sind sechs weitere hinzugekommen. Das Problem ließe sich (etwas künstlich) zuspitzen, indem man auch die Beinahe-Kollisionen auswertet, bei denen das Ständemehr klar und das Volksmehr knapp gescheitert ist. ${ }^{81}$ Insgesamt wird man die Diskussion aber eher unter die theoretischen Probleme verbuchen können.

\section{Abstimmungspraxis}

\section{a) Einbürgerungsinitiative (1. Juni 2008)}

Die Initiative „für demokratische Einbürgerungen“, die eine endgültige Entscheidung über das Bürgerrecht durch beliebige Gemeindeorgane ermöglichen wollte, ist bei nur 36,2 \% Ja-Stimmen deutlich abgelehnt worden (neuer Art. 38 Abs. 4 BV; eingereicht am 18 . November $2005 ; . . . / v i / v i s 320 . h t m l)$. Schon vor Einreichung der Initiative hatte es politische Aufregung gegeben, denn mit nur 100.038 gültigen Un-

79 Germann, Staatsreform (Fn. 62), S. 135 ff. (136 f.).

80 Übersicht mit Analyse bei Germann, Staatsreform (Fn. 62), S. 140; aktualisierte Übersicht der Bundeskanzlei unter .../va/vab_2_2_4_4.html.

81 Germann, Staatsreform (Fn. 62), S. 141 f.: insgesamt acht zwischen 1874 und 1990. 
terschriften wäre sie fast nicht zustande gekommen. Das wurde von der (rechtsnationalen) Schweizerischen Volkspartei (SVP), die die Initiative lanciert hatte, auf Verzögerungen bei der Ausstellung von Stimmrechtsbescheinigungen in den Wohngemeinden zurückgeführt. Ausschlaggebend für die klare Ablehnung dürfte gewesen sein, dass die Stimmenden sich sehr eng an die Abstimmungsparole ihrer jeweiligen Partei gehalten haben. ${ }^{82}$ Gleichzeitig befand sich die SVP, nachdem ihr Bundesratskandidat (Christoph Blocher) nicht wieder gewählt worden war, zum Zeitpunkt der Abstimmung wegen der selbst gewählten parlamentarischen Oppositionsrolle in einer isolierten Position gegenüber den anderen Parteien. Und schließlich wurden die Einbürgerungen an der Urne von einer breiten Mehrheit als willkürlich angesehen. ${ }^{83}$

Inhaltlich stellt die Initiative eine direkte Reaktion auf zwei Urteile des Bundesgerichts vom 9. Juli 2003 dar, die wiederum nur vor dem Hintergrund des im internationalen Vergleich einmaligen Einbürgerungsrechts der Schweiz zu verstehen sind. Die Einbürgerung erfolgt hierzulande dreistufig auf Gemeinde-, Kantons- und Bundesebene, wobei die erste Entscheidung durch die Gemeinde getroffen wird. ${ }^{84}$ Mit wenigen Ausnahmen sehen die Kantone und Gemeinden vor, dass sie die Verleihung des Bürgerrechts selbst dann ablehnen können, wenn alle gesetzlichen Einbürgerungsvoraussetzungen erfüllt sind - mithin kein Rechtsanspruch auf Einbürgerung besteht. Je nach gemeindlicher Kompetenzordnung konnte die Ablehnung eines Einbürgerungsgesuchs durch den politischen Akt einer Urnenabstimmung ohne inhaltliche Begründung erfolgen. Dem stellte das Gericht entgegen, dass es sich bei Einbürgerungsentscheidungen nicht nur um politische Akte, sondern auch um Rechtsanwendungsakte handle, bei denen das Rechtsstaatsprinzip einen Rechtsschutz erfordere. ${ }^{85}$ Die Beschwerdeführer rügten nämlich nach Überzeugung des Gerichts berechtigterweise eine Diskriminierung wegen ihrer ethnisch-kulturellen Herkunft aus Ex-Jugoslawien. ${ }^{86}$ Bei Einbürgerungsentscheiden des Stimmvolks an der Urne kann die besondere Begründungspflicht nicht wahrgenommen werden, die

82 Isabelle Engeli/Anouk Lloren/Alessandro Nai, Analyse der eidgenössischen Volksabstimmung vom 1. Juni 2008, VOX-Analyse, herausgegeben von gfs.bern, 2008, S. 16.

83 Engeli/Lloren/Nai, Analyse (Fn. 82), S. 20.

84 Aktueller Überblick zum inzwischen revidierten Einbürgerungsrecht bei Yvo Hangartner, Grundsatzfragen der Einbürgerung nach Ermessen, in: ZBl. 2009, S. 293-314 (293 ff.), allerdings mit der problematischen Annahme (S. 299, 301) eines fortbestehenden ,reinen“ (politischen) Ermessens auf Gesetzesebene, das dann durch Verfassungsprinzipien nachträglich korrigiert wird.

85 Früh bereits Georg Müller, Reservate staatlicher Willkür - Grauzonen zwischen Rechtsfreiheit, Rechtsbindung und Rechtskontrolle, in: Kurt Eichenberger (Hrsg.), FS Hans Huber, 1981, S. 109-125 (119f.).

86 BGE 129 I 217 E. 2 S. 223 ff. - Urnenabstimmung Emmen. Überblick zur Konzeption des Diskriminierungstatbestands in der schweizerischen Rechtsprechung und Lehre bei Walter Kälin/Martina Caroni, Das verfassungsrechtliche Verbot der Diskriminierung wegen der ethnisch-kulturellen Herkunft, in: Walter Kälin (Hrsg.), Das Verbot ethnisch-kultureller Diskriminierung. Verfassungs- und menschenrechtliche Aspekte, 1999, S. 67-94 (75 ff.). 
sich in solchen Fällen ergibt. ${ }^{87}$ In der Folge hatte das Bundesgericht mehrfach Gelegenheit, seine Rechtsprechung weiter zu konsolidieren, so dass Urnenabstimmungen über Einbürgerungsgesuche effektiv unmöglich wurden. ${ }^{88}$

Weil die kritischen Einbürgerungsfälle jeweils Ausdruck von Diskriminierung waren, hätte die Initiative die Rückkehr zu einer völkerrechtswidrigen Praxis bedeutet, denn in der Schweiz ist seit 1994 das Internationale Übereinkommen zur Beseitigung jeder Form von Rassendiskriminierung in Kraft. ${ }^{89}$ Da aber nur ein Verstoß gegen zwingende Bestimmungen des Völkerrechts die Ungültigerklärung zur Folge hat (Art. 194 Abs. 2 BV), musste die Volksabstimmung durchgeführt werden und hätte im Erfolgsfall zu einer verfassungsunmittelbaren Ausnahme vom Rechtsstaatsgebot und Diskriminierungsverbot geführt und gleichzeitig in einem unauflösbaren Widerspruch zur völkerrechtlichen Verpflichtung gestanden. Die unmittelbar an das Völkerrecht gebundenen Behörden hätten das Initiativanliegen darum selbst im Erfolgsfall nach schweizerischer Rechtspraxis nicht wirksam umsetzen dürfen. ${ }^{90}$

\section{b) Maulkorbinitiative (1. Juni 2008)}

Eine geradezu exemplarisch deutliche Ablehnung erfuhr die Initiative „Volkssouveränität statt Behördenpropaganda“, die dem Bundesrat und der Bundesverwaltung während des Abstimmungskampfes einen Maulkorb erteilen wollte und für dieses Anliegen nur 24,8 \% Ja-Stimmen erhielt (neuer Art. 34 Abs. 3 und 4 BV; eingereicht am 11. August 2004; .../vi/vis313.html). Erfolgreich war der indirekte Gegenvorschlag des Parlaments, mit dem statt eines Zurückhaltungsgebots ein Informationsgebot für den Bundesrat im Bundesgesetz über die politischen Rechte eingeführt wurde (.../as/2009/1.pdf). Die Abstimmung bildet damit den Endpunkt eines längerfristigen Meinungswechsels in Rechtsprechung und Lehre. Das politische Grundrecht auf Abstimmungs- und Wahlfreiheit, das inhaltlich den Anspruch auf unverfälschten Ausdruck des Volkswillens umfasst, sollte nach früherer Rechtsprechung und Lehre vor allem eine Zurückhaltung der staatlichen Behörden zur Folge haben. Während des Abstimmungskampfs durften Regierungsmitglieder nur noch ausnahmsweise tätig werden, insbesondere um irreführende Propaganda richtigzustel-

87 BGE 129 I 232 E. 3 S. 234 ff. - „Einbürgerungen vors Volk!“; aus der Literatur z.B. Regina Kiener, Rechtsstaatliche Anforderungen an Einbürgerungsverfahren, in: recht 2000, S. 213-225 (215 ff., 220); Andreas Auer/Nicolas von Arx, Direkte Demokratie ohne Grenzen? Ein Diskussionsbeitrag zur Frage der Verfassungsmässigkeit von Einbürgerungsbeschlüssen durch das Volk, in: AJP 2000, S. 923-935 (930 f.).

88 BGE 130 I 140 - Gemeindebürgerrechtsverordnung Schwyz; BGE 132 I 196 - Einbürgerung Burg.

89 Auer/Tornay, Limites (Fn. 68), S. $741 \mathrm{f}$.

90 Auer/Tornay, Limites (Fn. 68), S. 745 ff. Zum allgemeinen Problem der völkerrechtswidrigen Volksinitiativen siehe oben S. 219. 
len. ${ }^{91}$ Demgegenüber hat die jüngere Rechtsprechung vorsichtig eine „Beratungsfunktion" innerhalb des politischen Grundrechts verortet und die Literatur sogar eine allgemeine Informationspflicht aus ihm abgeleitet. ${ }^{92}$ Das Volk hat nun deutlich gemacht, dass es sich durch bundesrätliche Medienauftritte während eines Abstimmungskampfes keinesfalls entmachtet vorkommt, sondern im Gegenteil ein Interesse an diesen zusätzlichen Stellungnahmen hat. Außerdem billigt eine deutliche Mehrheit der Stimmenden dem Bundesrat den Anspruch zu, seine Meinung öffentlich darlegen zu können. ${ }^{93}$

\section{c) Unverjährbarkeitsinitiative (30. November 2008)}

Zur allgemeinen Überraschung errang die Initiative „für die Unverjährbarkeit pornografischer Straftaten an Kindern" das doppelte Mehr von Volk (51,9 \%) und Ständen (16 4/2). In der Bundesverfassung führt sie zu folgender Ergänzung, die sofort mit dem Abstimmungstermin in Kraft tritt (Art. 15 Abs. 3 BPR):

Art. 123 b Unverjährbarkeit der Strafverfolgung und der Strafe bei sexuellen und bei pornografischen Straftaten an Kindern vor der Pubertät.

Die Verfolgung sexueller oder pornografischer Straftaten an Kindern vor der Pubertät und die Strafe für solche Taten sind unverjährbar.

Die Initiative war am 1. März 2006 eingereicht (.../vi/vis329.html) und von der Bundesversammlung zur Ablehnung empfohlen worden, weil die strafrechtliche Verjährung ohnehin mehrfach verlängert worden war, so dass zuletzt eine Strafklage mindestens bis zum vollendeten 25 . Lebensjahr des Opfers eingereicht werden konnte. Zusätzlich unternahm das Parlament in einem indirekten Gegenvorschlag eine weitere Ausdehnung, die im Ergebnis eine Geltendmachung der Strafbarkeit bis zum 33. Lebensjahr bedeutet hätte. Trotz aller Moderation stimmte das Volk für die radikale Regelung; die emotionalen Argumente errangen hier offenbar den Vorrang vor juristischen und politisch-abwägenden Überlegungen.$^{94}$ Ein ebenfalls äußerst emotionales Nachspiel hatte die Umsetzung des neuen Artikels, bei der das Justizdepartement nicht von einer Rückwirkung ausgegangen ist, woraufhin ein enttäuschtes Missbrauchsopfer sich unter dramatischen Umständen das Leben nahm.

91 Vgl. BGE 108 Ia 155 E. 3 b S. 157 f. - Umfahrung Eglisau, m.w.N. Aus der Literatur beispielsweise Stephan Widmer, Wahl- und Abstimmungsfreiheit, 1989, S. 178 ff., 185 ff., m.w.N.; zuletzt nochmals Hansjörg Seiler, Auf dem Weg zur gelenkten Demokratie? Behördliche Intervention in die politische Meinungsbildung, Oder: Was bedeutet politische Führung?, in: Peter Hänni (Hrsg.), FS Thomas Fleiner, 2003, S. 573-591 (577 ff.).

92 BGE 130 I 290 E. 3.2 S. 294 - Zürcher Anwaltsverband; in der Literatur insbesondere Michel Besson, Behördliche Information vor Volksabstimmungen. Verfassungsrechtliche Anforderungen an die freie Willensbildung der Stimmberechtigten in Bund und Kantonen, 2003, S. 154 ff., m.w.N.

93 Engeli/Lloren/Nai, Analyse (Fn. 82), S. $28 \mathrm{f}$.

94 Vgl. Oliver Krömler/Thomas Milic/Bianca Rousselot, Analyse der eidgenössischen Abstimmung vom 30. November 2008, VOX-Analyse, herausgegeben von gfs.bern, 2009, S. 12. 
Die konkrete Anpassung des Strafrechts wird schon aus diesem Grunde, aber auch wegen der generalklauselartigen Formulierung (,,vor der Pubertät“), die Bundesversammlung intensiv beschäftigen.

Sowohl in der Thematik als auch im Verlauf weist die Unverjährbarkeitsinitiative Gemeinsamkeiten mit der Verwahrungsinitiative von 2004 auf. ${ }^{95}$ Trotz einhelliger Ablehnung durch Parlamentarier und Strafrechtler nahmen Volk und Stände damals die Initiative nach einer emotional geführten Debatte an. Die Regelung, dass eine Überprüfung der lebenslänglichen Verwahrung nur bei neuen wissenschaftlichen Erkenntnissen zulässig sein soll, gilt zudem als Verstoß gegen die Vorschrift der Europäischen Menschenrechtskonvention, nach der ein Recht auf Überprüfung des Freiheitsentzugs innerhalb kurzer Frist möglich sein muss (Art. 5 Abs. 4 EMRK). Da die Konventionsregelungen aber nicht insgesamt zum zwingenden Völkerrecht zählen, musste die Initiative gleichwohl für gültig erklärt werden. Im Umsetzungsprozess wurde dann entgegen dem Protest der Initiantinnen und entgegen dem Wortlaut der neuen Verfassungsbestimmung doch die Freilassung aus individuellen (statt objektiv-wissenschaftlichen) neuen Erkenntnissen ermöglicht. Da sich diese Regelungen im Strafgesetzbuch finden, also in einem Bundesgesetz, lässt sich eine Durchsetzung des genauen Initiativtextes vor dem Bundesgericht nicht erzwingen (vgl. Art. 190 BV).

\section{d) Hanfinitiative (30. November 2008)}

Erwartungsgemäß scheiterte die Initiative „Für eine vernünftige Hanf-Politik mit wirksamem Jugendschutz" bei nur 36,7\% Ja-Stimmen deutlich (neuer Art. 105 a BV; eingereicht am 13. Januar 2006; .../vi/vis325.html). Sie wollte Konsum, Besitz, Erwerb und Anbau von Betäubungsmittelhanf (Cannabis) für den Eigenbedarf straffrei erklären. Aus Sicht des Bundesrats läge in einer derart weitgehenden Entkriminalisierung ein Verstoß gegen die von der Schweiz ratifizierten internationalen Übereinkommen im Drogenbereich. ${ }^{96}$ Die Bundesversammlung stellte der Initiative ihre seit längerem geplante Revision des Betäubungsmittelgesetzes gegenüber, nach der die Straflosigkeit vorgesehen ist, wenn nur „eine geringfügige Menge eines Betäubungsmittels für den eigenen Konsum vorbereitet"wird (Art. 19 b BetmG ${ }^{97}$ ). Die Hanfinitiative hatte in dieser Abstimmung ursprünglich relativ gute Erfolgsaussichten, weil zwei große Parteien (SP, FDP) eine Parole zu ihrer Unterstützung ausgegeben hatten. Das deutliche Scheitern resultierte aus dem Umstand, dass die An-

95 Eidgenössische Volksinitiative „Lebenslange Verwahrung für nicht therapierbare, extrem gefährliche Sexual- und Gewaltstraftäter“; neuer Art. 123 a BV; Annahme am 8. Februar 2004 mit 56,2 \% Ja-Stimmen und 19 5/2 Ständen; .../vi/vis294.html. Die Behandlungsfrist dieser kontroversen Initiative war zuvor um ein Jahr verlängert worden.

96 Botschaft zur Volksinitiative ,für eine vernünftige Hanf-Politik mit wirksamem Jugendschutz“ vom 15. Dezember 2006, in: BB1 2007245 (248) = .../ff/2007/245.pdf.

97 Bundesgesetz über die Betäubungsmittel und die psychotropen Stoffe vom 3. Oktober 1951 (Betäubungsmittelgesetz, BetmG, SR 812.121 = .../sr/8/812.121.de.pdf). 
hänger dieser Parteien diesmal nach individuellen Wertehaltungen und nicht nach Parolen stimmten. ${ }^{98}$ Dabei stand das Jugendschutzmotiv im Vordergrund. ${ }^{99}$

Die Revision des Betäubungsmittelgesetzes, die den indirekten Gegenvorschlag zur Hanfinitiative bildete, war ihrerseits wegen der darin enthaltenen Liberalisierungsschritte so umstritten, dass das Referendum ergriffen wurde, welches ebenfalls am 30. November zur Abstimmung kam. Nachdem die Legalisierung der therapeutischen Heroinabgabe mehrfach bei Volksabstimmungen gescheitert war, gelang es diesmal, sie als Teile eines zunächst projektweise erprobten Viersäulenmodells der Drogenpolitik dauerhaft auf eine gesetzliche Grundlage zu stellen. ${ }^{100}$

\section{e) Referendum gegen Personenfreizügigkeit (8. Februar 2009)}

Überraschend deutlich sprach sich das Volk für die Erweiterung der Personenfreizügigkeit auf Bulgarien und Rumänien aus (59,6 \% Ja-Stimmen) ${ }^{101}$ Hier hatte die Bundesversammlung nach langer öffentlicher Diskussion eine Verknüpfung von zwei Beschlussteilen vorgenommen: die unbefristete Weiterführung des Freizügigkeitsabkommens einerseits und die Ausdehnung dieses Freizügigkeitsabkommens auf Bulgarien und Rumänien andererseits. Zwischen beiden bestand zwar ein sachlicher Zusammenhang, doch umstritten war nur der Erweiterungsteil. Der Bundesrat hatte in seiner Vorlage noch eine gemeinsame Botschaft für zwei getrennte Beschlüsse vorgesehen, die es dem Volk ermöglicht hätten, getrennt gegen jeden Teil vorzugehen. Indem die Mehrheit der Bundesversammlung die Materien später zu einem Beschlusspaket verschnürte, konnten die Gegner der Erweiterung nur das Referendum gegen beide Teile ergreifen, was sie als eine demokratisch und rechtsstaatlich unhaltbare Verkürzung ihres Referendumsrechts rügten. Nach dem Verlauf der öffentlichen Diskussion war nicht auszuschließen, dass das Volk aus Protest gegen die Paketlösung dem Referendum zustimmt. Das ist indes nicht geschehen. Auf der Basis eines vergleichsweise guten Informationsstandes vertrauten die Stimmenden der Einschätzung des Bundesrats, dass die Schweiz gar keine andere Wahl

98 Krömler/Milic/Rousselot, Analyse (Fn. 94), S. 29 f.

99 Krömler/Milic/Rousselot, Analyse (Fn. 94), S. 37.

100 68,1 \% stimmten für die Revision: Änderung des Bundesgesetzes über die Betäubungsmittel und die psychotropen Stoffe (Betäubungsmittelgesetz, BetmG) vom 20. März 2008, in: BB1 $20082269=\ldots$.. ff / 2008 / 2269.pdf; dazu der vorausgegangene Bericht der Kommission für soziale Sicherheit und Gesundheit des Nationalrates vom 4. Mai 2006, in: BB1 20068573 $=\ldots / \mathrm{ff} / 2006 / 8573$. pdf, sowie die Stellungnahme des Bundesrates zu diesem Bericht vom 29. September 2006, in: BB1 $20068645=\ldots /$ ff / 2006 / 8645.pdf. Chronologie unter .../ cr / 2006 / 20061652.html.

101 Bundesbeschluss über die Genehmigung der Weiterführung des Freizügigkeitsabkommens zwischen der Schweiz und der Europäischen Gemeinschaft und ihren Mitgliedstaaten sowie über die Genehmigung und die Umsetzung des Protokolls über die Ausdehnung des Freizügigkeitsabkommens auf Bulgarien und Rumänien vom 13. Juni 2008, in: BB1 20085323 $=\ldots / \mathrm{ff} / 2008 / 5323$. pdf. Dazu die vorausgegangene Botschaft des Bundesrats vom 14. März 2008, in: BBl $20082135=\ldots /$ ff/2008/2135.pdf. Chronologie unter .../cr/2008/200806 53.html. 
habe, wenn sie den bisher erfolgreichen Weg der Europapolitik durch bilaterale Verträge weiter beschreiten wolle. ${ }^{102}$

\section{f) Referendum gegen biometrische Pässe (17. Mai 2009)}

Mit dem äußerst geringen Abstand von nur 5504 Stimmen und damit einem der knappsten Ergebnisse seit Bestehen der ersten Bundesverfassung (1848) siegten die Befürworter (50,1\%) des biometrischen Passes. Einen vergleichbar knappen Abstimmungsausgang hatte es zuletzt im November 2002 zur Asylinitiative gegeben (Differenz: 3422 Stimmen, Nachzählung 4208 Stimmen). Das Passreferendum hatte in Inhalt und Verfahren einige Besonderheiten. Es richtete sich auf einen Bundesbeschluss zur Umsetzung des weiterentwickelten Schengen-Besitzstandes. ${ }^{103}$ Insoweit waren allerdings beide Seiten einig, dass es der Einführung eines biometrischen Passes bedurfte, um den Schengenregeln zukünftig genügen zu können. Umstritten war indes, dass die Bundesversammlung über den Schengen-Acquis hinaus eine zentrale Speicherung der Fingerabdrücke im geänderten Ausweisgesetz vorsah und den Bürgern nicht die Wahl eines biometriefreien Passes ließ. Dadurch wird sich in Zukunft eine Sammlung der Fingerabdrücke aller Schweizerinnen und Schweizer ergeben, die zwar bisher nicht zur Strafverfolgung eingesetzt werden darf, zukünftige Weiterungen der Zweckbindung aber sehr vereinfacht. Eine vertiefte Auseinandersetzung über die zentrale Datenspeicherung hatte im Parlament nicht stattgefunden. Die nötige Diskussion wurde im Rahmen des Abstimmungskampfes auch nur ansatzweise nachgeholt, denn bis zuletzt waren die technischen Details der Realisierung offen, obgleich gerade von ihnen eine realistische Einschätzung der Missbrauchsgefahren abhängt.

Der Referendumsprozess war insofern neuartig, als erstmals die Jungparteien von links bis rechts mit den Mitteln des Social Networking (Facebook) eine gemeinsame Front gegen das Parlament gebildet haben. Entsprechend ergab sich auch keine schlüssige Abstimmungsgeographie nach Sprachgrenze oder Stadt-Land-Gefälle. Das Ständemehr, auf das es mangels Verfassungsänderung nicht ankam, wurde zudem deutlich verfehlt, weil 16 Kantone mehrheitlich mit Nein stimmten - ein weiteres Anzeichen für den denkbar knappen Abstimmungsausgang. Wären die Biometriepässe in Form einer Volksinitiative verlangt worden, hätten sie mangels Ständemehrs keinen Erfolg gehabt. Gleichzeitig wäre dies dann eines der seltenen Beispiele für ein Auseinanderfallen von Volksmehr und Ständemehr gewesen.

102 Hans Hirter/Wolf Linder, Analyse der eidgenössischen Abstimmung vom 8. Februar 2009, VOX-Analyse, herausgegeben von gfs.bern, 2009, S. 13, 15, 16 f., $21 \mathrm{ff}$.

103 Bundesbeschluss über die Genehmigung und die Umsetzung des Notenaustauschs zwischen der Schweiz und der Europäischen Gemeinschaft betreffend die Übernahme der Verordnung (EG) Nr. 2252/2004 über biometrische Pässe und Reisedokumente (Weiterentwicklung des Schengen-Besitzstands) vom 13. Juni 2008, in: BB1 2008, 5309 = .../ff/2008/5309.pdf. Dazu die vorausgegangene Botschaft des Bundesrats vom 18. Juni 2007, in: BB1 $20075159=\ldots /$ ff/2007/5159.pdf. Chronologie unter .../cr/2007/20070681.html. 


\section{g) Weitere Volksabstimmungen}

Nachdem die Volksinitiative „Für tiefere Krankenkassenprämien in der Grundversicherung", welche auf mehr Wettbewerb und einen reduzierten Leistungskatalog abzielte (neuer Art. 117 a BV; eingereicht am 28. Juli 2004; .../vi/vis312.html), im Januar 2008 zurückgezogen worden war, ist nun auch der direkte Gegenvorschlag der Bundesversammlung, mit dem die wesentlichen Grundsätze des Krankenpflegesystems (Konkurrenz, Transparenz, Qualität, Wirtschaftlichkeit) in die Verfassung geschrieben werden sollten (geänderter Art. 117 BV, neuer Art. 117 a BV; beschlossen am 21. Dezember 2007; www.admin.ch/ch/d/pore/rf/cr/2005/200511 08.html), mit nur 30,5\% Ja-Stimmen sehr deutlich abgelehnt worden.

Die Volksinitiative „Für ein flexibles AHV-Alter“ wollte unter bestimmten Bedingungen eine Option für die Pensionierung ab dem 62. Altersjahr einführen (neuer Art. 112 Abs. 2 Bst. e BV; eingereicht am 28. März 2006; .../vi/vis336.html). Der Bundesrat erachtete die Neufassung der 11. AHV-Revision mit ihrer breiteren Flexibilisierungsmöglichkeit und der Vorruhestandsleistung für den unteren Mittelstand als indirekten Gegenvorschlag. ${ }^{104} \mathrm{Er}$ hatte damit Erfolg: die Initiative wurde mit nur 41,4\% Ja-Stimmen vom Volk verworfen.

Die Volksinitiative „Verbandsbeschwerderecht: Schluss mit der Verhinderungspolitik - Mehr Wachstum für die Schweiz!“ strebte den Ausschluss des Verbandsbeschwerderechts bei Entscheiden des Volkes oder von Parlamenten an (neuer Art. 30 a BV; eingereicht am 11. Mai 2006; .../vi/vis333.html). Das Anliegen war Ausdruck einer Empörung über verzögerte Stadionbauten und konnte somit auf einen Populismusbonus hoffen. Gleichwohl lehnte das Volk die Initiative mit einem Nein-Stimmen-Anteil von $66 \%$ deutlich ab.

Dem vom Parlament vorgelegten direkten Gegenvorschlag zur im Oktober 2008 zurückgezogenen Volksinitiative „Ja zur Komplementärmedizin“, welche die umfassende Berücksichtigung der Komplementärmedizin anstrebte (neuer Art. 118 a BV; eingereicht am 15. September 2005; .../vi/vis331.html), stimmte das Volk am 17. Mai 2009 mit $67 \%$ Ja-Stimmen überaus deutlich zu. Der Gegenentwurf sah bloß eine (nicht umfassende) Berücksichtigung der Komplementärmedizin vor (neuer Art. 118 a BV; beschlossen am 3. Oktober 2008; www.admin.ch/ch/d/pore/va/ 20090517/det541.html). Interessanterweise wird das klare Ergebnis zunächst keine Folgen haben, denn für die Einbeziehung der fünf verlangten alternativen Heilmethoden in die Grundversicherung (Pflanzenheilkunde, Neuraltherapie, Homöopathie, chinesische Arznei-Therapie, anthroposophische Medizin) müssen weitere Regeln erlassen werden, welche Einzelmethoden aufgrund welchen wissenschaftlichen Nachweises aufgenommen werden sollen.

104 Geschäft Nr. 05.093 - 11. AHV-Revision (Neufassung) unter .../geschaefte.aspx?gesch_id= 20050093. 


\section{Hängige Volksinitiativen und Referenden}

\section{a) Initiierungsphase}

Während der Unterschriftensammlung lassen sich die im Rahmen der Vorprüfung genehmigten Unterschriftenlisten auf den Aktualitätsseiten der Bundeskanzlei abrufen (www.bk.admin.ch/aktuell/abstimmung/vi/). In diesem frühen Stadium befinden sich derzeit (Juni 2009)

- die Volksinitiative „68 Milliarden für die soziale Sicherheit“, welche als Reaktion auf die finanziellen Beiträge an die UBS zusätzliche 68 Milliarden für die soziale Sicherheit (AHV, IV, Erwerbsersatzordnung und Arbeitslosenversicherung) fordert (neuer Art. 114 a BV; Sammelfrist bis 26. November 2010; .../vi/ vis373.html),

- die Volksinitiative „zum Schutz vor Passivrauchen“, die ein grundsätzliches Verbot des Rauchens in allen öffentlich zugänglichen Innenräumen einführen will (neuer Art.118a BV; Sammelfrist bis 19. November 2010; .../vi/ vis371.html),

- die Volksinitiative „Verteidigen wir die Schweiz! Das Bankgeheimnis muss in die Bundesverfassung“, mit der die Lockerung des Bankgeheimnisses durch den Bundesrat (März 2009) zurückgenommen werden soll (neuer Art. 13 Abs. 3 bis 5 BV; Sammelfrist bis 1 . Oktober $2010 ; . . . / v i /$ vis369.html),

- die Volksinitiative „Für den öffentlichen Verkehr“, welche die Förderung des öffentlichen Verkehrs erstmals in der Bundesverfassung verankert und die Neuverteilung von Mitteln vorschlägt (neue Art. 81 a, 86 Abs. 3ter und $5 \mathrm{BV}$, geänderte Art. 86 Abs. 3 und 4 BV; Sammelfrist bis 17. September 2010; .../vi/ vis366.html),

- die Volksinitiative „Für Geldspiele im Dienste des Gemeinwohls“, mit der die Verwendung von Erträgen aus Geldspielen für das Gemeinwohl (Sport, Kultur und Soziales) konkretisiert werden soll (geänderter Art. 106 BV, neue Art. 106 a und 106 b BV; Sammelfrist bis 22. Oktober 2009; .../vi/vis364.html),

- die Volksinitiative „Für die Stärkung der Volksrechte in der Außenpolitik (Staatsverträge vors Volk!)“،, welche verlangt, dass völkerrechtliche Verträge in wichtigen Bereichen zwingend dem Volk und den Kantonen zur Abstimmung unterbreitet werden und eine doppelte Mehrheit (Volks- und Ständemehr) erreichen müssen (neuer Art. 140 Abs. 1 Bst. d BV; Sammelfrist bis 4. September $2009 ; . . . / v i /$ vis363.html),

- die Volksinitiative „6 Wochen Ferien für alle“, die die Erhöhung des gesetzlichen Mindestferienanspruchs fordert (neuer Art. 110 Abs. 4 BV; Sammelfrist bis 15 . Juli $2009 ;$.../vi/vis362.html). 
Im Sammelstadium gescheitert sind

- die Volksinitiative „Für freie Meinungsäußerung - weg mit dem Maulkorb!“, die den Rassendiskriminierungstatbestand des Strafrechts (Art. 261 bis StGB) abschaffen wollte (neuer Art. 16 Abs. 4 BV; Sammelfrist bis 7. Februar 2009; .../ $\mathrm{vi} / \mathrm{vis} 360 . \mathrm{html})$,

- die Volksinitiative „Prävention statt Abzockerei - Für eine Neuausrichtung der Tabaksteuer (Tabakinitiative)“, welche die Senkung der Tabaksteuersätze auf $20 \%$ des Kleinhandelspreises sowie die Verwendung von Tabaksteuererträgen für die Tabakprävention forderte (neuer Art. 131 Abs. 4 BV; Sammelfrist bis 12 . Juni $2008 ; . . . / v i / v i s 350 . h t m l)$.

\section{b) Botschaftsphase}

Bereits eingereicht, aber mangels Botschaft noch beim Bundesrat hängig (.../vi/ vis_1_3_1_2.html), sind derzeit

- die Volksinitiative „Gegen neue Kampfflugzeuge“, welche ein zehnjähriges Beschaffungsmoratorium fordert (neuer Art. 197 Ziff. 8 BV; eingereicht am 8. Juni $2009 ; . . . / v i / v i s 365 . h t m l)$,

- die Volksinitiative „Für den Schutz vor Waffengewalt“, die Armeewaffen nur noch in gesicherten Räumen aufbewahrt wissen will (neuer Art. 118 a BV; zustande gekommen am 16. März 2009; .../vi/vis361.html),

- die Volksinitiative „Eigene vier Wände dank Bausparen“, welche die Förderung des selbstgenutzten Wohneigentums anstrebt (neuer Art. 108 a BV; zustande gekommen am 17. Februar 2009; .../vi/vis358.html),

- die Volksinitiative „Sicheres Wohnen im Alter“, mit der Menschen im Pensionsalter bei selbstgenutztem Wohneigentum steuerlich entlastet werden sollen (neuer Art. 108 b BV; zustande gekommen am 17. Februar 2009; .../vi/vis359. html),

- die Volksinitiative ,jugend + musik“, welche eine Verbesserung der musikalischen Bildung von Kindern und Jugendlichen im obligatorischen Schulunterricht, Unterstützung von Ausbildungen an Musikschulen sowie die Förderung von musikalisch Begabten anstrebt (neuer Art. 67 a BV; zustande gekommen am 21. Januar 2009; .../vi/vis355.html),

- die Volksinitiative „Für ein steuerlich begünstigtes Bausparen zum Erwerb von selbst genutztem Wohneigentum und zur Finanzierung von baulichen Energiespar- und Umweltschutzmaßnahmen (Bauspar-Initiative)“, die das im Kanton Basel-Landschaft praktizierte (und mittlerweile vom Steuerharmonisierungsgesetz nicht mehr zugelassene) Bausparmodell auf nationaler Ebene einführen sowie Hauseigentümern bei energiesparenden Sanierungsmaßnahmen fördern will (neue Art. 129 a und 129 b BV; zustande gekommen am 29. Oktober 2008; .../ vi/vis352.html), 
- die Volksinitiative „Für menschenfreundlichere Fahrzeuge (Stopp-OffroaderInitiative)“, welche die Reduktion der Umweltbelastung sowie den besseren Schutz der Verkehrsteilnehmer durch einen Verkaufsstopp bestimmter Neuwagen und eine Geschwindigkeitsbeschränkung für Altfahrzeuge erreichen will (neuer Art. 82 a BV; zustande gekommen am 8. September 2008; .../vi/vis351. html),

- die Volksinitiative „Raum für Mensch und Natur (Landschaftsinitiative)“, die unter anderem die Vergrößerung des Baugebiets während 20 Jahren verbieten will (geänderter Art. 75 BV; zustande gekommen am 27. August 2008; .../vi/ vis356.html),

- die Volksinitiative „Für ein gesundes Klima“, welche bis im Jahr 2020 eine mindestens 30-prozentige Reduktion der Treibhausgasemissionen durch effizientere Nutzung der Energie sowie durch Förderung von erneuerbaren Energien vorsieht (neuer Art. 89 a BV; zustande gekommen am 2. April 2008; .../vi/vis354.html),

- die Volksinitiative „Für die Ausschaffung krimineller Ausländer (Ausschaffungsinitiative)“, die den Verlust des Aufenthaltsrechts von Ausländern vorsieht, welche wegen bestimmter Straftaten rechtskräftig verurteilt worden sind oder welche missbräuchlich Leistungen von Sozialwerken bezogen haben (neuer Art. 121 Abs. 3-6 BV; zustande gekommen am 7. März 2008; .../vi/vis357.html).

Besonders hervorzuheben sind unter diesen die Ausschaffungsinitiative (www.ausschaffungsinitiative.ch), die analog zur SD-Initiative zum zweiten Fall einer Ungültigerklärung werden könnte, sowie die Offroader-Initiative (www.stoppoffroader.ch, www.menschenfreundlicher.ch), weil sie trotz der erheblichen Eingriffe in die persönliche Entscheidungsfreiheit nach Umfragen eine hohe Zustimmung genießt.

\section{c) Beratungsphase}

Vom Bundesrat mit einer Botschaft und Ablehnungsempfehlung versehen und jetzt bei der Bundesversammlung hängig (.../vi/vis_1_3_1_3.html) sind derzeit

- die Volksinitiative „Für faire Steuern. Stopp dem Missbrauch beim Steuerwettbewerb (Steuergerechtigkeits-Initiative)“, welche die kantonale Steuerautonomie und den kantonalen Steuerwettbewerb einschränken will (neuer Art. 129 Abs. $2^{\text {bis }}$ BV; Botschaft vom 6. März 2009 ohne Gegenvorschlag; .../vi/vis349.html),

- die Volksinitiative „Gegen die Abzockerei“, nach der die Grundsätze für die Lohnpolitik in einer Aktiengesellschaft zukünftig durch die Generalversammlung festgelegt werden sollen (neuer Art. 95 Abs. 3 BV; Botschaft vom 5. Dezember 2008 mit indirektem Gegenvorschlag; .../vi/vis348.html),

- die Volksinitiative „Gegen maßlosen Bau umwelt- und landschaftsbelastender Anlagen“, die belastende bauliche Anlagen nur noch zulassen will, wenn ge- 
samtschweizerisch ein dringendes Bedürfnis dafür besteht (neuer Art. 75 Abs. 4 BV; Botschaft vom 29. Oktober 2008 ohne Gegenvorschlag; .../vi/vis344.html),

- die Volksinitiative „Schluss mit uferlosem Bau von Zweitwohnungen!“, welche die Beschränkung des Zweitwohnungsanteils pro Gemeinde auf höchstens $20 \%$ der Bruttogeschossfläche fordert (neuer Art. 75 a BV; Botschaft vom 29. Oktober 2008 ohne Gegenvorschlag; .../vi/vis345.html),

- die Volksinitiative „Gegen Tierquälerei und für einen besseren Rechtsschutz der Tiere (Tierschutzanwalt-Initiative)“, welche unter anderem die Institution eines Tierschutzanwaltes vorsieht (neuer Art. 80 Abs. 4 und 5 BV; Botschaft vom 14. Mai 2008 ohne Gegenvorschlag; .../vi/vis340.html),

- die Volksinitiative „Lebendiges Wasser (Renaturierungs-Initiative)“, welche die Wiederherstellung naturnaher Verhältnisse von Fließgewässern fordert (neuer Art. 76 a BV; Botschaft vom 27. Juni 2007 ohne Gegenvorschlag; .../vi/vis335. html).

Besonders erwähnenswert ist unter diesen die Abzockerinitiative, der die Bundesversammlung im indirekten Gegenvorschlag nicht sehr stark entgegengekommen ist, obgleich die Initiative sowohl in der Bevölkerung als auch in Wirtschaftkreisen erhebliche Zustimmung genießt.

\section{d) Abstimmungsphase}

Von der Bundesversammlung in ihrer Schlussabstimmung am 12. Juni 2009 zur Ablehnung empfohlen und damit abstimmungsreif sind

- die Volksinitiative „Für ein Verbot von Kriegsmaterial-Exporten“, welche die Ausfuhr von Waffen verbieten will (neue Art. 107 Abs. 3 und 107 a BV; Botschaft vom 27. August 2008 ohne Gegenvorschlag; .../vi/vis346.html),

- die Volksinitiative „Gegen den Bau von Minaretten“, welche die Errichtung von Minaretten absolut verbieten will (neuer Art. 72 Abs. 3 BV; Botschaft vom 27. August 2008 ohne Gegenvorschlag; .../vi/vis353.html).

Besonders erwähnenswert ist unter diesen die Minarettinitiative (www.minarette.ch), die selbst ohne eine Annahme erhebliche Unruhe auslösen wird. Gerade im Ausland, wo die hiesige Praxis im Detail nicht bekannt ist, wird kaum zu vermitteln sein, warum in der Schweiz eine Abstimmung über eindeutig völkerrechtswidrige Ziele geführt werden darf.

\section{Direkte Demokratie in den Kantonen}

Anders als im Bund lässt sich für Kantone und erst recht für Gemeinden nicht sinnvoll eine Gesamtübersicht der Volksabstimmungen eines Berichtsjahres erstellen. Selbst die bisher von Politikwissenschaftlern erarbeiteten Datenbanken werden üb- 
licherweise nicht nachgeführt, weil der Aufwand dafür zu groß wäre. Sofern für einzelne Zeitabschnitte Daten vollständig erfasst wurden, ergeben sich daraus im Durchschnitt etwa 110 kantonale Einzelabstimmungen pro Jahr. ${ }^{105}$

\section{Grundsätzliche Fragen}

$\mathrm{Zu}$ den Charakteristika der kantonalen im Gegensatz zu den eidgenössischen Abstimmungen gehört, dass gleichartige direktdemokratische Instrumente eine höhere Erfolgschance aufweisen als auf Bundesebene: im Durchschnitt hat jede vierte Initiative und jedes zweite fakultative Referendum Erfolg. ${ }^{106}$ Bei den Initiativen ist die Erfolgsaussicht auf Kantonsebene (25\%) folglich etwa zweieinhalb mal so groß wie auf Bundesebene (10\%).

Ein wiederkehrendes Sachproblem der kantonalen Abstimmungspraxis ist deren Verhältnis zum Bundesrecht, wird es doch häufig als unbefriedigend angesehen, wenn sich das Kantonsvolk zu einer Entscheidung durchgerungen hat, die dann durch eine Diskussion über bundeseinheitliche Regelungen wieder in Frage gestellt wird. So werden die kantonalen Neuregelungen der Hundehaltung, die nach mehreren Todesfällen mit Kampfhunden durchgeführt wurden, überlagert von einer Gesetzesdiskussion in der Bundesversammlung. Die Medikamentenabgabe durch Ärzte, über die in Zürich während der vergangenen zehn Jahre dreimal abgestimmt wurde, zuletzt im November 2008 mit einer knappen Befürwortung (53,7 \%), könnte gemäß den aktuellen Vorschlägen des Bundesrats im Rahmen der Revision des Heilmittelgesetzes verboten werden. Und die in Bergkantonen umstrittenen Begrenzungen des Zweitwohnungsbaus könnten durch eine Initiative auf Bundesebene überholt werden.

\section{Abstimmungspraxis}

Im Berichtszeitraum sind auf kantonaler Ebene gehäuft Abstimmungen über das Schulwesen durchgeführt worden. Deren Hintergrund ist zunächst die Harmonisierung der obligatorischen Schule Schweiz (HarmoS), die als interkantonales Konkordat den vom Volk vor drei Jahren mit sehr deutlicher Mehrheit ( $86 \%$ ) revidierten Bildungsartikel der Bundesverfassung (Art. 62 Abs. 4 BV) umsetzen will. Mit Wirkung ab 2014 sollen dazu die Strukturen und Ziele der kantonalen Schulsysteme angeglichen werden. Besonders umstritten ist die Verlängerung des Schulobligatoriums auf elf Jahre und seine Vorverlegung auf das vierte Altersjahr, wodurch die bisher freiwilligen Kindergartenzeiten zu Eingangsstufen der Schulzeit mutieren. Die für das Inkrafttreten erforderliche Mindestzustimmung durch zehn Kantone ist

105 Errechnet aus den Angaben bei Vatter, Kantonale Demokratien (Fn. 21), S. 268 ff. mit Tabelle 34: mehr als 2000 Abstimmungen im Zeitraum 1980 bis 1997. 
inzwischen erreicht, doch liegt dies überwiegend an denjenigen Kantonen, bei denen das Parlament entschieden hat, ohne dass es zum Referendum kam. Unter den bisherigen Volksabstimmungen zum HarmoS-Konkordat zeigt sich ein anderes Bild: von sechs durchgeführten Abstimmungen führten nur zwei zur Annahme, vier dagegen zur Ablehnung. In mindestens vier weiteren Kantonen wird noch abgestimmt.

Im Schulwesen sind außerdem auch die kantonsspezifischen Revisionen einer ständigen Kritik und dementsprechend häufigen Referendumsabstimmungen ausgesetzt. Ausgelöst durch das überraschend schlechte Abschneiden der Schweiz im internationalen Vergleichstest Pisa 2000 (etwa $15 \%$ der Schülerinnen und Schüler können nach neun Schuljahren kaum lesen) geht derzeit eine Revisionswelle durch die kantonalen Schulsysteme. Neben der Einführung von Blockzeiten und Ganztagesstrukturen gehört dabei vor allem der Fremdsprachenunterricht zu den kontroversen Themen, weil hierbei die bisher üblicherweise unterrichtete zweite Landessprache (Französisch, Deutsch) unter den Druck des Englischunterrichts gerät und vereinzelt sogar Frühenglisch angestrebt wird. Insgesamt zeigt sich im Volk eine eher konservative Grundhaltung gegenüber den Reformanliegen. So wurden die zuvor abgeschafften Primarschulnoten ab der vierten Klasse im Kanton Appenzell Ausserrhoden mit klarer Mehrheit (67 \% Ja-Stimmen) gegen Regierung und Parlament durchgesetzt. Das Volk im Aargau verwarf mit jeweils deutlichen Mehrheiten alle fünf Reformvorhaben der kantonalen Bildungspolitiker und beendete damit gleichzeitig die Bestrebungen nach einem gemeinsamen Bildungsraum mit Solothurn und Basel. Ähnlich erging es den beiden Teilen der Bildungsreform in Schaffhausen. Die Graubündner lehnten die Abschaffung des Religionsunterrichts mit klarer Mehrheit ab (69,2\%). Außergewöhnlich deutlich (21\% Ja-Stimmen) scheiterte schließlich eine Volksinitiative im Kanton Basel-Landschaft, mit der Eltern zur Verwirklichung der freien Schulwahl gefordert hatten, dass Privatschulen pro Schülerin oder Schüler ebenso viele Mittel erhalten sollten wie öffentliche Schulen. Statt dessen wird nun der Gegenvorschlag umgesetzt, nach dem die kantonalen Zuschüsse für anerkannte Privatschulen von 2000 auf 2500 Franken erhöht werden. Weil gleichlautende Initiativen in anderen Kantonen lanciert wurden, dürfte dieses deutliche Ergebnis überregional Beachtung finden.

Volksabstimmungen über Kampfhundeverbote waren in zwei Kantonen erfolgreich (Genf, Zürich). In zahlreichen anderen Kantonen wurden zwar keine absoluten Verbote angenommen, wohl aber strenge Auflagen (Maulkorb- und Leinenpflicht) sowie eine Bewilligungspflicht für das Halten von Hunden mit erhöhtem Gefährdungspotential.

Mit der Abschaffung der Pauschalbesteuerung (52,9\% Ja-Stimmen) ist in Zürich bereits eine Entscheidung gefallen, die in anderen Kantonen noch bevorsteht. Bei dieser Steuerart wird reichen Ausländern als Gegenleistung für die erwerbstätigkeitslose Wohnsitznahme im Kanton ein individuelles Angebot unterbreitet, das die sonst das Steuerrecht dominierende Gleichbehandlung unterläuft. Die Ratio der Regelung liegt im Gewinn für die jeweilige Gemeinde, die solche Ausländer sonst gar nicht als Steuerzahler einbeziehen könnte; die öffentliche Kritik richtet sich auf den insofern als unfair empfundenen Steuerwettbewerb und auf die als gleichheits- 
widrig angesehene Sonderbehandlung. Die effektive Ungleichbehandlung wird nämlich mit einer trickreichen allgemeinverbindlichen Regelung bewirkt: statt Einkommens- und Vermögenssteuer zahlen reiche Ausländer ihre Abgabe auf der Basis der „Lebenshaltungskosten“. Als Premiere gilt, dass mit dieser Abstimmung ein Kanton erstmalig ein Steuerprivileg wieder abgeschafft hat. Zürich ist allerdings wegen der sehr geringen Zahl an Pauschalbesteuerten (137) und dem ansonsten relativ robusten Steueraufkommen viel weniger von dieser zusätzlichen Einkommensquelle abhängig als einzelne Kantone in der Westschweiz, so dass sich die Abstimmungsfrage für das dortige Volk etwas anders stellen wird. Dass in Zürich die Alternative Liste (AL) als sehr kleine Linksaußen-Partei mit dieser Volksinitiative erfolgreich sein konnte, liegt wahrscheinlich auch an der seit drei Jahren von 10.000 auf 6.000 Unterschriften gesenkten Eintrittshürde. Hinsichtlich der Bundessteuer existieren die Privilegien weiterhin, sind allerdings einer stärker werdenden Kritik ausgesetzt und werden zunächst im Parlament zu Diskussionen führen.

Ein Rauchverbot in Restaurants ist inzwischen bei fast allen Kantonen erfolgreich eingeführt worden. Zuvor hatte das Bundesgericht zur Genfer Volksinitiative festgehalten, dass sie gültig sei, weil der Bundesgesetzgeber im Arbeitsschutz nicht abschließend legiferiert habe. ${ }^{107}$ Die Abstimmungsvorlagen beziehungsweise die diese abmildernden Gegenvorschläge sehen in der Regel die Ausnahme eines sogenannten „Fumoirs“ vor: das sind abgetrennte Räume mit wirksamer Belüftung, in denen keine Bedienung erfolgen darf. Im Grundsatz erklären die Kantone das Rauchen aber in allen öffentlich zugänglichen Räumen für verboten. Einen etwas anderen Weg gehen die ländlichen Kantone, die zusätzliche Ausnahmen für kleine Landgaststätten gestatten.

Bei den Hooligan-Kontrollinstrumenten hat sich im Berichtszeitraum eine teilweise Kompetenzverlagerung ergeben. Waren im Rahmen der Fußballeuropameisterschaft 2008 und der Eishockeyweltmeisterschaft 2009 neben der unbefristeten Hooligan-Datenbank auch noch befristete Polizeikompetenzen durch Bundesgesetz eingeführt worden, ${ }^{108}$ so sind jetzt dieselben, von der Polizei geschätzten Eingriffsbefugnisse durch ein Hooligan-Konkordat zwischen den Kantonen abgelöst worden. Das Volk in Luzern hat die weitgehenden Kontrollrechte dieses Konkordates mit einer überwältigenden Mehrheit gutgeheißen.

Polizeigesetzliche Wegweisungsartikel sind nach Bern und St. Gallen nunmehr auch in Luzern und Basel-Stadt durch Volksabstimmung eingeführt worden - und zwar mit unzweideutigen Mehrheiten (77,9\% und 78,9\% Ja-Stimmen). Das Instrument richtet sich gegen die hierzulande sogenannten „Randständigen“, die im öffentlichen Raum, insbesondere rund um Bahnhöfe oder (in Luzern) vor dem daneben

107 BGE 133 I 110 E. 4 S. 115 ff. - Passivrauchen Genf; deutsche Übersetzung in: Pra 2007 Nr. 123, S. 829-844.

108 Änderung des Bundesgesetzes über Massnahmen zur Wahrung der inneren Sicherheit (BWIS, SR $120=\ldots /$ sr / $1 /$ 120.de.pdf) vom 24. März 2006, AS $20063703=\ldots /$ as $/ 2006 /$ 3703.pdf; die Befristung der neuen Ingerenzregeln zum Schutz bei Sportveranstaltungen erfolgte bis zum 31. Dezember 2009 und bezog sich auf Rayonverbote, Meldeauflagen und Polizeigewahrsam (Art. 24 b, 24 d, 24 e BWIS). 
gelegenen Kultur- und Kongresszentrum, selbst ohne Gewalttätigkeit oder Lärmverursachung als Belästigung empfunden werden. Im zeitlichen Ausmaß variieren die Polizeirechtsartikel allerdings stark; das Spektrum reicht für einfache Belästigungsfälle von 24 Stunden (Luzern) über 72 Stunden (Basel-Stadt) bis hin zu mehreren Monaten (Bern).

Bei der umstrittenen Einbürgerungspraxis, die den Gemeinden eine Urnenabstimmung mangels Entscheidungsbegründung unmöglich macht, ${ }^{109}$ hat das Stimmvolk in St. Gallen nach langer politischer Blockade jetzt eine Kompromisslösung gutgeheißen (65,3\% Ja-Stimmen). Künftig werden primär Einbürgerungsräte in den Gemeinden über die Einbürgerung entscheiden, wobei allen Stimmberechtigten ein Anfechtungsrecht zusteht. Nach schriftlicher und begründeter Anfechtung gelangt das Einbürgerungsgesuch zum Letztentscheid an die Bürgerversammlung. Damit wird eine Urnenabstimmung wieder ermöglicht, allerdings nur auf der Grundlage einer inhaltlichen Begründung, die wiederum eine gerichtliche Kontrollmöglichkeit eröffnet und damit voraussichtlich eine diskriminierungsfreie Praxis bewirkt.

Prominent, aber überwiegend chancenlos sind in den Kantonen der Schweiz die Initiativen zur Senkung des Stimmrechtsalters auf 16 Jahre, wie dies in Österreich bereits Praxis ist. In den Verfassungsrevisionen der letzten Jahre hat sich die Senkung des Stimmrechts nicht durchsetzen lassen. Eine Ausnahme bildet insofern der Kanton Glarus, in dem das Begehren erfolgreich war. Eine entsprechende Volksinitiative im Kanton Uri ist hingegen mit sehr geringem Befürworteranteil (20,1 \% JaStimmen) gescheitert, ebenso wie zuvor im Kanton Basel-Stadt (28 \% Ja-Stimmen). Im Kanton Bern wird im November 2009 darüber abgestimmt werden; in Graubünden ist eine entsprechende Initiative lanciert.

\section{Direkte Demokratie in den Gemeinden}

\section{Grundsätzliche Fragen}

Der inhaltliche Umfang der gemeindlichen Volksabstimmungen variiert kantonal mit dem unterschiedlichen Umfang der gemeindlichen Kompetenzen. Die Gemeindeautonomie, die in der Schweiz traditionell als kantonales verfassungsmäßiges Recht qualifiziert wird, findet sich in der Bundesverfassung nur ,nach Massgabe des kantonalen Rechts“" garantiert (Art. 50 Abs. $1 \mathrm{BV}$ ), hängt also davon ab, inwieweit ein Kanton seinen Gemeinden eigene Regelungsspielräume belässt und ihnen so eine „relativ erhebliche Entscheidungsfreiheit" einräumt. ${ }^{110}$ Im Ergebnis gibt es gleichwohl viele Gemeinsamkeiten, denn alle Kantone orientieren sich an dem Ziel, die Kompetenzen so dezentral wie möglich zuzuweisen (Subsidiaritätsprinzip).

Zu den wichtigsten Zuständigkeiten der Gemeinden zählen die Einbürgerung, das lokale Raumordnungsrecht, die Veranstaltungsregeln für Märkte, die Einrichtung lokaler Behörden und Regelung ihres Verfahrens, das Personalrecht der Gemeinde,

109 Zur Einbürgerungsinitiative siehe oben S. 223.

110 BGE 122 I 279 E. 8 b S. 290 - Parkierungsgebühren Zürich, st. Rspr. 
die Denkmalpflege und Kulturförderung, das örtliche Schulwesen, die Gemeindepolizei, das Bestattungswesen, die Gemeindefinanzen, insbesondere die Liegenschaftssteuer als häufige direkte Gemeindesteuer, der Baumschutz, die Gemeindestraßen einschließlich der Regelung des ruhenden Verkehrs (Parkierreglement), die Einrichtungen des öffentlichen Verkehrs, die Abwasser- und Abfallentsorgung, die örtliche Wohnungsbauförderung, die Einrichtung von Kindertagesstätten, die lokale Feuerwehr sowie die Zulassung von Taxis. Innerhalb dieser Materien finden Volksabstimmungen besonders häufig über die Revision von Polizei- und Steuergesetzen sowie über Raumordnungsfragen statt, beispielsweise bei Neufestsetzung von Baulinien und Umzonung von Flächen. Besonders häufig sind schließlich die kommunalen Abstimmungen über den Auf- und Ausbau von Infrastruktureinrichtungen größter Vielfalt - etwa Bahnhöfe, Hallenbäder, Gemeindehäuser, Seilbahnen, Skiarenen, Feuerwehrgebäude, Schulhäuser, Kulturzentren, Grünanlagen, Kindertagesstätten, Spitäler, Pflegezentren, Parkhäuser und Straßenbahnverbindungen („Tramstrecken"). Im Infrastrukturbereich wird teilweise sogar über den Austausch der Straßenbeleuchtung per Volksabstimmung entschieden: so im Berichtszeitraum in der Stadt Luzern über den „Plan Lumière“, der nach einer freiwilligen Kürzung durch den Stadtrat letztlich angenommen wurde (57,8 \% Ja-Stimmen).

\section{Abstimmungspraxis}

Besondere Aufmerksamkeit erfahren die kommunalen Volksabstimmungen in den großen Städten, weil diese auf andere Gemeinden ausstrahlen - entweder direkt durch überregionale Wirkung oder indirekt, indem sie einen Trend setzen, dem andere Gemeinden folgen. Im Berichtszeitraum hat beispielsweise das Volk in der Stadt Luzern eine Sportarena mit Fußballstadion beschlossen, deren Dimensionen (Gesamtkosten 250 Millionen Franken) bereits von einem überregionalen Einzugsgebiet ausgeht. Die Stadt Zürich hat als erste schweizerische Gemeinde und mit überraschender Deutlichkeit (76,4\% Ja-Stimmen) beschlossen, langfristig aus der Atomenergie auszusteigen und die Reduktion des Kohlendioxidausstoßes in der Gemeindeordnung (Verfassung) festzuschreiben. Noch deutlicher (80,4\% Ja-Stimmen) fiel die Abstimmung über die Finanzierung von Windenergieanlagen aus.

Ebenfalls zu den Materien der Volksabstimmung auf Gemeindeebene gehören die (sehr häufigen) Annahmen neuer Gemeindeordnungen und die früher sehr seltenen Gemeindefusionen, die in der Schweiz unter anderem wegen der Ämterbesetzungsnot neuerdings häufiger vorkommen. Im Berichtszeitraum war die Fusionsabstimmung in drei Tessiner Gemeinden erfolgreich, die gemeinsam die neue Gemeinde Centovalli gründeten. Gelungen sind außerdem zwei Gemeindefusionen im Kanton Aargau. Gescheitert ist hingegen eine Fusion im Broyetal (Kanton Waadt), weil drei von zwölf Gemeinden den Zusammenschluss ablehnten. Gescheitert ist außerdem der bisher größte Fusionsversuch im Kanton Bern, weil zwei der sechs beteiligten Gemeinden das Zusammengehen ablehnten. Prominent und bisher weitgehend erfolgreich ist das Vorhaben der Stadt Luzern, die durch Eingliederung von 
Umlandgemeinden bis 2016 von 59.000 auf 150.000 Einwohner wachsen und damit zur viertgrößten Stadt der Schweiz aufsteigen möchte. Nach einer schon beschlossenen Teilfusion hat das Volk in drei weiteren Gemeinden rund um Luzern jetzt teils deutlich, teils knapp Ja zu einer Verhandlungsaufnahme gesagt, während sich eine vierte, die durch lokale Steuersenkungen heraussticht, bisher verweigerte.

Eine Stufe unterhalb der Fusion bewegt sich die Einrichtung von Regionalkonferenzen. Eine solche hatte der Kanton Bern bereits in der Region Interlaken erfolgreich etabliert. Die Stimmberechtigten von 100 Gemeinden stimmten nun einer weiteren Konferenz in der Region um die Stadt Bern zu. Mit solchen Konferenzen, die eine Entscheidungskompetenz der Gemeindepräsidenten in Planungs-, Kultur- und Verkehrsfragen begründet, wird die bisherige interkommunale Kooperation vereinfacht.

Zwischen Fusion und Regionalkonferenz ist die Agglomeration angesiedelt, zu der sich neun von zehn Gemeinden rund um die Stadt Freiburg nach jahrelangen Planungen im Wege der Volksabstimmung zusammengefunden haben. Die Besonderheit dieser „Agglo“ besteht darin, dass sie mit eigener Legislative und Exekutive ausgestattet ist und kommunale Kernaufgaben (Verkehr, Raumplanung, Umweltschutz, Wirtschaftsförderung, Kultur, Tourismus) übernimmt. Mangels Erfahrung mit derlei Zwischenformen ist noch unklar, ob das Konstrukt auf Dauer bestehen oder nur eine Vorstufe zur Fusion darstellen wird. Bezeichnenderweise sind die neun zustimmenden Gemeinden mehrheitlich französischsprachig, während die einzige deutschsprachige Gemeinde die Teilnahme mit deutlicher Mehrheit (60 \% NeinStimmen) abgelehnt hat.

In der Stadt Bern setzte sich mit der sehr deutlichen Annahme eines kommunalen Bettelverbots (75,8 \% Ja-Stimmen), bei dem gleichzeitig noch , ungebührliches Verhalten" unter Bußgeldandrohung gestellt wird, an der Urne ein Trend fort, der gegenwärtig jedweder Law-and-order-Regelung breite Zustimmung beschert. Zur Abstimmung gestellt wurden diese Regelungen im Rahmen eines Bahnhofsreglements für die städtischen Flächen rund um den frisch renovierten Bahnhofsplatz. Dieser sollte mit dem Gesetzgebungsakt wieder zur „Visitenkarte“ für die Bundesstadt gemacht werden.

\section{Abgekürzte Links:}

$\ldots /$ as $/=$ www.admin.ch/ch/d/as/

.../ff/ = www.admin.ch/ch/d/ff/

.../geschaefte.aspx $=$ www.parlament.ch/d/suche/seiten/geschaefte.aspx

.../ref/ = www.bk.admin.ch/aktuell/abstimmung/ref/

$\ldots / \mathrm{cr} /=$ www.admin.ch $/ \mathrm{ch} / \mathrm{d} /$ pore $/ \mathrm{rf} / \mathrm{cr} /$

.../sr/ = www.admin.ch $/ \mathrm{ch} / \mathrm{d} / \mathrm{sr} /$

.../va/ = www.admin.ch/ch/d/pore/va/

.../vi/ = www.admin.ch/ch/d/pore/vi/ 